\title{
Electronics and optoelectronics of two-dimensional transition metal dichalcogenides
}

\author{
Qing Hua Wang ${ }^{1}$, Kourosh Kalantar-Zadeh ${ }^{2}$, Andras Kis ${ }^{3}$, Jonathan N. Coleman ${ }^{4,5}$ and Michael S. Strano ${ }^{1 \star}$
}

\begin{abstract}
The remarkable properties of graphene have renewed interest in inorganic, two-dimensional materials with unique electronic and optical attributes. Transition metal dichalcogenides (TMDCs) are layered materials with strong in-plane bonding and weak out-of-plane interactions enabling exfoliation into two-dimensional layers of single unit cell thickness. Although TMDCs have been studied for decades, recent advances in nanoscale materials characterization and device fabrication have opened up new opportunities for two-dimensional layers of thin TMDCs in nanoelectronics and optoelectronics. TMDCs such as MoS $_{2}$, $\mathrm{MoSe}_{2}, \mathrm{WS}_{2}$ and $\mathrm{WSe}_{2}$ have sizable bandgaps that change from indirect to direct in single layers, allowing applications such as transistors, photodetectors and electroluminescent devices. We review the historical development of TMDCs, methods for preparing atomically thin layers, their electronic and optical properties, and prospects for future advances in electronics and optoelectronics.
\end{abstract}

M any two-dimensional (2D) materials exist in bulk form as stacks of strongly bonded layers with weak interlayer attraction, allowing exfoliation into individual, atomically thin layers ${ }^{1}$. The form receiving the most attention today is graphene, the monolayer counterpart of graphite. The electronic band structure of graphene has a linear dispersion near the $\mathrm{K}$ point, and charge carriers can be described as massless Dirac fermions, providing scientists with an abundance of new physics ${ }^{2,3}$. Graphene is a unique example of an extremely thin electrical and thermal conductor ${ }^{4}$, with high carrier mobility ${ }^{5}$, and surprising molecular barrier properties ${ }^{6,7}$.

Many other 2D materials are known, such as the TMDCs ${ }^{8,9}$, transition metal oxides including titania- and perovskite-based oxides $^{10,11}$, and graphene analogues such as boron nitride $(\mathrm{BN})^{12,13}$. In particular, TMDCs show a wide range of electronic, optical, mechanical, chemical and thermal properties that have been studied by researchers for decades ${ }^{9,14,15}$. There is at present a resurgence of scientific and engineering interest in TMDCs in their atomically thin $2 \mathrm{D}$ forms because of recent advances in sample preparation, optical detection, transfer and manipulation of 2D materials, and physical understanding of 2D materials learned from graphene.

The 2D exfoliated versions of TMDCs offer properties that are complementary to yet distinct from those in graphene. Graphene displays an exceptionally high carrier mobility exceeding $10^{6} \mathrm{~cm}^{2} \mathrm{~V}^{-1} \mathrm{~s}^{-1}$ at $2 \mathrm{~K}$ (ref. 16) and exceeding $10^{5} \mathrm{~cm}^{2} \mathrm{~V}^{-1} \mathrm{~s}^{-1}$ at room temperature for devices encapsulated in $\mathrm{BN}$ dielectric lay$\mathrm{ers}^{5}$; because pristine graphene lacks a bandgap, however, fieldeffect transistors (FETs) made from graphene cannot be effectively switched off and have low on/off switching ratios. Bandgaps can be engineered in graphene using nanostructuring ${ }^{17-19}$, chemical functionalization ${ }^{20}$ and applying a high electric field to bilayer graphene $e^{21}$, but these methods add complexity and diminish mobility. In contrast, several 2D TMDCs possess sizable bandgaps around 1-2 eV (refs 9,14), promising interesting new FET and optoelectronic devices.

TMDCs are a class of materials with the formula $\mathrm{MX}_{2}$, where $\mathrm{M}$ is a transition metal element from group IV ( $\mathrm{Ti}, \mathrm{Zr}$, Hf and so on), group V (for instance V, Nb or Ta) or group VI (Mo, W and so on), and $\mathrm{X}$ is a chalcogen ( $\mathrm{S}, \mathrm{Se}$ or Te). These materials form layered structures of the form $\mathrm{X}-\mathrm{M}-\mathrm{X}$, with the chalcogen atoms in two hexagonal planes separated by a plane of metal atoms, as shown in Fig. 1a. Adjacent layers are weakly held together to form the bulk crystal in a variety of polytypes, which vary in stacking orders and metal atom coordination, as shown in Fig. 1e. The overall symmetry of TMDCs is hexagonal or rhombohedral, and the metal atoms have octahedral or trigonal prismatic coordination. The electronic properties of TMDCs range from metallic to semiconducting, as summarized in Table 1 . There are also TMDCs that exhibit exotic behaviours such as charge density waves and superconductivity ${ }^{22-24}$, but are beyond the scope of the present review.

The layer-dependent properties of TMDCs have recently attracted a great deal of attention. For example, in several semiconducting TMDCs there is a transition from an indirect bandgap in the bulk to a direct gap in the monolayer: for $\mathrm{MoS}_{2}$ the bulk indirect bandgap of $1.3 \mathrm{eV}$ increases to a direct bandgap of $1.8 \mathrm{eV}$ in single-layer form ${ }^{25}$. The direct bandgap also results in photoluminescence from monolayer $\mathrm{MoS}_{2}$, which opens the possibility of many optoelectronic applications $s^{25}$. The electronic structure of $\mathrm{MoS}_{2}$ also enables valley polarization, which is not seen in bilayer $\mathrm{MoS}_{2}$ (refs 26-28). In general, there are many interesting layer-dependent properties in 2D materials, including graphene and TMDCs, which differ greatly from the properties of the bulk materials.

Although TMDCs have been widely studied for decades, their role as near-atomically thin materials is new, and this Review aims to introduce readers to what is known and to suggest possibilities for many exciting developments.

\section{Synthesis}

Reliable production of atomically thin, 2D TMDCs with uniform properties is essential for translating their new electronic and optical properties into applications. Here we review the available methods for top-down exfoliation from bulk materials and for bottom-up synthesis, and evaluate their relative merits.

'Department of Chemical Engineering, Massachusetts Institute of Technology, Cambridge, Massachusetts 02139-4307, USA, ${ }^{2}$ School of Electrical and Computer Engineering, RMIT University, Melbourne, Victoria, Australia, ${ }^{3}$ Electrical Engineering Institute, École Polytechnique Fédérale de Lausanne (EPFL), CH-1015 Lausanne, Switzerland, ${ }^{4}$ School of Physics, Trinity College Dublin, Dublin 2, Ireland, ${ }^{5}$ Centre for Research on Adaptive Nanostructures and Nanodevices (CRANN), Trinity College Dublin, Dublin 2, Ireland. *e-mail: strano@mit.edu 




b

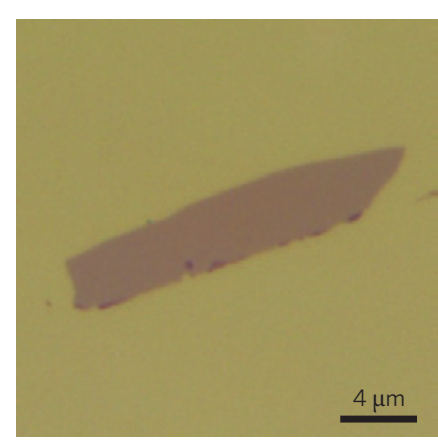

c



d

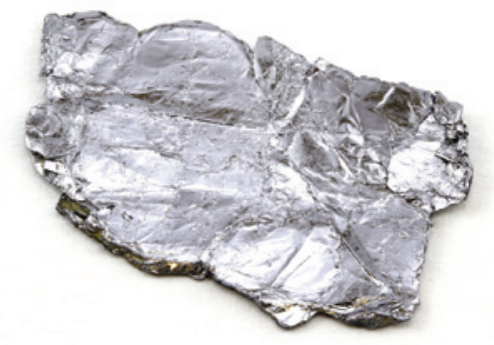

e

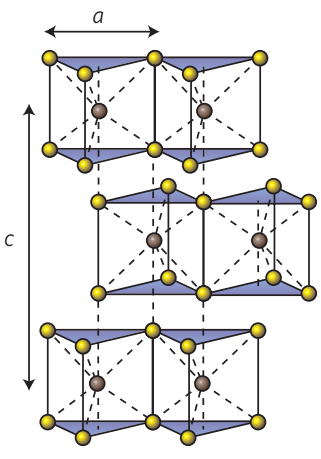

$2 \mathrm{H}$

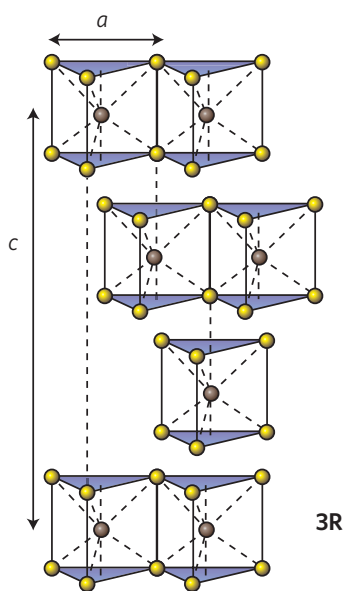
$3 R$ $\circ \mathrm{X}$

O $M$

Figure 1 | Structure of TMDC materials. a, Three-dimensional schematic representation of a typical $\mathrm{MX}_{2}$ structure, with the chalcogen atoms $(X)$ in yellow and the metal atoms $(M)$ in grey. $\mathbf{b}, \mathbf{c}$, Monolayer flake of $\mathrm{MoS}_{2}$. Optical microscopy image (b) and atomic force microscopy (AFM) image (c). d, Photograph of bulk $\mathrm{MoS}_{2}$ crystal, which is approximately $1 \mathrm{~cm}$ long. $\mathbf{e}$, Schematics of the structural polytypes: $2 \mathrm{H}$ (hexagonal symmetry, two layers per repeat unit, trigonal prismatic coordination), 3R (rhombohedral symmetry, three layers per repeat unit, trigonal prismatic coordination) and $1 T$ (tetragonal symmetry, one layer per repeat unit, octahedral coordination). The chalcogen atoms $(\mathrm{X})$ are yellow and the metal atoms (M) are grey. The lattice constants $a$ are in the range 3.1 to $3.7 \AA$ for different materials ${ }^{83}$. The stacking index $c$ indicates the number of layers in each stacking order, and the interlayer spacing is $\sim 6.5 \AA$. Figure reproduced with permission from: a, ref. 34, (c) 2011 NPG; b,c, ref. 36, (c) 2011 IOP.

Top-down methods. Atomically thin flakes of TMDCs can be peeled from their parent bulk crystals by micromechanical cleavage using adhesive tape $e^{1,29-35}$, applied to substrates and optically identified by light interference ${ }^{36,37}$, using the same techniques that were developed for graphene. A bulk crystal of $\mathrm{MoS}_{2}$ is shown in Fig. 1d, and a monolayer flake of $\mathrm{MoS}_{2}$ derived from micromechanical cleavage is shown in Fig. 1b,c. Other layered materials such as $\mathrm{BN}$ (refs $1,12,29$ ) and oxide nanosheets ${ }^{10,38}$ can also be mechanically exfoliated into single sheets by this method. Mechanical cleavage produces single-crystal flakes of high purity and cleanliness that are suitable for fundamental characteriza$\operatorname{tion}^{30-33}$ and for fabrication of individual devices ${ }^{11,31,32,34,35,39-41}$. This method is not scalable, however, and does not allow systematic control of flake thickness and size. Recently, a focused laser spot has been used to thin $\mathrm{MoS}_{2}$ down to monolayer thickness by thermal ablation with micrometre-scale resolution, but the requirement for laser raster scanning makes it challenging for scale-up ${ }^{42}$.

For obtaining large quantities of exfoliated nanosheets, liquidphase preparations of TMDCs are very promising. They permit additional applications such as composites and hybrids by simple mixing of dispersions of different materials ${ }^{43,44}$, and thin films and coatings by filtration, inkjet printing, spray coating and doctor blading. Because solution-based graphene has been used to make highfrequency flexible electronics with a current gain cut-off frequency of $2.2 \mathrm{GHz}$ (ref. 45), we expect that solution-based TMDCs will have similarly good prospects for making flexible electronics and composite materials.

The intercalation of TMDCs by ionic species ${ }^{46-51}$ allows the layers to be exfoliated in liquid. The intercalation method was first demonstrated in the 1970s (ref. 51) and the subsequent exfoliation into thin layers by Morrison, Frindt and co-workers in the 1980s (ref. 47), and these methods are experiencing renewed interest today $^{49,50}$. The intercalation of TMDCs by lithium was demonstrated in the 1970s (ref. 51), and the intercalation-driven exfoliation was first advanced by Morrison, Frindt and co-workers ${ }^{47}$. The typical procedure involves submerging bulk TMDC powder in a solution of a lithium-containing compound such as $n$-butyllithium for more than a day to allow lithium ions to intercalate, followed by exposing the intercalated material to water. The water reacts vigorously with the lithium between the layers to evolve $\mathrm{H}_{2}$ gas, which rapidly separates the layers ${ }^{47,49}$. Solution-phase $\mathrm{MoS}_{2}$ flakes derived from lithium-based intercalation and exfoliation are shown in Fig. $2 b$.

Such chemical exfoliation methods produce gram quantities of submicrometre-sized monolayers ${ }^{52}$, but the resulting exfoliated material differs structurally and electronically from the bulk mate$\mathrm{rial}^{49}$. In particular, for $\mathrm{MoS}_{2}$ the process changes the electronic structure of the exfoliated nanosheets from semiconducting to metallic, and the Mo atom coordination is changed from trigonal prismatic $\left(2 \mathrm{H}-\mathrm{MoS}_{2}\right)$ to octahedral $\left(1 \mathrm{~T}-\mathrm{MoS}_{2}\right)^{49,53-55}$. Annealing at $300{ }^{\circ} \mathrm{C}$ can cause a phase change from $1 \mathrm{~T}-\mathrm{MoS}_{2}$ to $2 \mathrm{H}-\mathrm{MoS}_{2}$, restoring the Mo atom coordination, and restoring the semiconducting 
Table 1 | Summary of TMDC materials and properties.

\begin{tabular}{|c|c|c|c|c|c|c|}
\hline & \multicolumn{2}{|c|}{$-S_{2}$} & \multicolumn{2}{|c|}{$-\mathrm{Se}_{2}$} & \multicolumn{2}{|c|}{$-\mathrm{Te}_{2}$} \\
\hline & $\begin{array}{l}\text { Electronic } \\
\text { characteristics }\end{array}$ & References & $\begin{array}{l}\text { Electronic } \\
\text { characteristics }\end{array}$ & References & $\begin{array}{l}\text { Electronic } \\
\text { characteristics }\end{array}$ & References \\
\hline $\mathrm{Ta}$ & $\begin{array}{l}\text { Metal; } \\
\text { superconducting; } \\
\text { CDW }\end{array}$ & $138,164(E)$ & $\begin{array}{l}\text { Metal; } \\
\text { superconducting; } \\
\text { CDW }\end{array}$ & 138,164 (E) & Metal & $83(T)$ \\
\hline \multirow[t]{2}{*}{ Mo } & $\begin{array}{l}\text { Semiconducting } \\
1 \mathrm{~L}: 1.8 \mathrm{eV}\end{array}$ & $31(E)$ & $\begin{array}{l}\text { Semiconducting } \\
1 \mathrm{~L}: 1.5 \mathrm{eV}\end{array}$ & $82(T)$ & $\begin{array}{l}\text { Semiconducting } \\
1 \mathrm{~L}: 1.1 \mathrm{eV}\end{array}$ & $82(T)$ \\
\hline & Bulk: $1.2 \mathrm{eV}$ & $88(E)$ & Bulk: $1.1 \mathrm{eV}$ & $88(E)$ & Bulk: $1.0 \mathrm{eV}$ & $165(E)$ \\
\hline W & $\begin{array}{l}\text { Semiconducting } \\
1 \mathrm{~L}: 2.1 \mathrm{eV}\end{array}$ & $25(T)$ & $\begin{array}{l}\text { Semiconducting } \\
1 \mathrm{~L}: 1.7 \mathrm{eV}\end{array}$ & $83(T)$ & $\begin{array}{l}\text { Semiconducting } \\
1 \mathrm{~L}: 1.1 \mathrm{eV}\end{array}$ & $83(T)$ \\
\hline
\end{tabular}

The electronic characteristic of each material is listed as metallic, superconducting, semiconducting or charge density wave (CDW). For the semiconducting materials, the bandgap energies for monolayer (1L) and bulk forms are listed. The cited references are indicated as experimental (E) or theoretical (T) results.

bandgap of the pristine material, as evidenced by the re-emergence of bandgap photoluminescence ${ }^{49}$. Lithium-based chemical exfoliation has been demonstrated ${ }^{55,56}$ for TMDCs such as $\mathrm{MoS}_{2}, \mathrm{WS}_{2}$, $\mathrm{MoSe}_{2}$ and $\mathrm{SnS}_{2}$.

An alternative method of lithiation that is faster and more controllable uses an electrochemical cell with a lithium foil anode and TMDC-containing cathode, as recently demonstrated by Zeng et al. ${ }^{50,57}$. Because the intercalation occurs while a galvanic discharge is occurring in the electrochemical cell, the degree of lithiation can be monitored and controlled. The resulting Li-intercalated material is exfoliated by sonication in water as before, yielding monolayer TMDC nanosheets. The method was first demonstrated for $\mathrm{MoS}_{2}$, $\mathrm{WS}_{2}, \mathrm{TiS}_{2}, \mathrm{TaS}_{2}, \mathrm{ZrS}_{2}$ and graphene ${ }^{50}$, and then for $\mathrm{BN}, \mathrm{NbSe}_{2}, \mathrm{WSe}_{2}$, $\mathrm{Sb}_{2} \mathrm{Se}_{3}$ and $\mathrm{Bi}_{2} \mathrm{Te}_{3}$ (ref. 57). This method requires only several hours for $\mathrm{Li}$ intercalation, compared with more than a day for the n-butyllithium method.

Alternatively, TMDCs can be exfoliated by ultrasonication in appropriate liquids, including organic solvents, aqueous surfactant solutions, or solutions of polymers in solvents ${ }^{43,44,58,59}$. Typically, ultrasonication results in the mechanical exfoliation of layered crystals to give flakes that are a few hundred nanometres in size. The exfoliated nanosheets are stabilized against re-aggregation either by solvation or by steric or electrostatic repulsion due to the adsorption of molecules from solution. Solutions of 2D layered materials exfoliated in organic solvents are shown in Fig. 2a, along with thin films from filtration of solution-based material ${ }^{43}$. Molecules from solution probably adsorb onto the TMDC nanosheets by non-covalent interactions, but more work is required to determine whether there is any effect on electronic properties.

The energy required to exfoliate layered crystals can be quantified from the surface energy, which is the energy to remove a monolayer from the crystal divided by twice the monolayer surface area. Experimental results derived from both exfoliation studies and inverse gas chromatography suggest that $\mathrm{BN}, \mathrm{MoS}_{2}, \mathrm{WS}_{2}$ and $\mathrm{MoSe}_{2}$ have surface energies in the range $65-75 \mathrm{~mJ} \mathrm{~m}^{-2}$ (refs 43,60 ). These values are comparable to the surface energy of graphene, which has been measured in the range $65-120 \mathrm{~mJ} \mathrm{~m}^{-2}$ (refs 61,62), and they indicate that inorganic layered compounds can be exfoliated as easily as graphite, or even more easily.

The main advantage of ion exfoliation is that it gives a high yield of monolayers ${ }^{49}$, and improvements in $\mathrm{Li}$ intercalation by an electrochemical method have made the process faster and more controllable ${ }^{50,57}$. But the flammability of the Li compounds under ambient conditions requires the work to be carried out under inert gas, and $\mathrm{Li}$ is an increasingly expensive resource, so there is incentive to find alternative intercalants. Liquid exfoliation is insensitive to ambient conditions, but it yields a relatively low concentration of monolayer flakes $^{43}$. Thus, for electronic or photonic applications where monolayers are required, well-annealed ion-exfoliated nanosheets will be useful; but for applications such as composite materials where large quantities are required, it is possible that liquid exfoliation will be preferred. Rational control over nanosheet thickness and size is likely to be required for nanoelectronics and in particular optoelectronics. Also by analogy with graphene, new layer-controlled chemistries $^{63}$ and post-synthesis sorting of flakes by layer thickness ${ }^{64}$ and lateral size $e^{65}$ may offer solutions.

Bottom-up methods. Developing methods for synthesizing largearea and uniform layers is an important step for applications such as wafer-scale fabrication of electronic devices and flexible, transparent optoelectronics. As previously demonstrated for graphene, the development of wafer-scale synthesis methods via chemical vapour deposition (CVD) on metal substrates ${ }^{66}$ and epitaxial growth on $\mathrm{SiC}$ substrates ${ }^{67}$ has enabled large-scale device fabrication ${ }^{68-70}$.

Some CVD methods for growing atomically thin films of $\mathrm{MoS}_{2}$ on insulating substrates have recently been reported ${ }^{71-73}$. These methods use different solid precursors heated to high temperatures: sulphur powder and $\mathrm{MoO}_{3}$ powder vaporized and co-deposited onto a nearby substrate ${ }^{71,74}$; a thin layer of Mo metal deposited onto a wafer heated with solid sulphur ${ }^{72}$; and substrates dip-coated in a solution of $\left(\mathrm{NH}_{4}\right)_{2} \mathrm{MoS}_{4}$ and heated in the presence of sulphur gas $^{73}$. These CVD-related methods are summarized in Fig. 2c-e. In many of these methods, the final $\mathrm{MoS}_{2}$ film thickness is dependent on the concentration or thickness of the initial precursor, although precise control of the number of layers over a large area has not yet been achieved. CVD growth of $\mathrm{MoS}_{2}$ has also been demonstrated using previously CVD-grown graphene on $\mathrm{Cu}$ foil as a surface template, resulting in single-crystal flakes of $\mathrm{MoS}_{2}$ several micrometres in lateral size ${ }^{75}$. These CVD reports are still relatively early results but hold promise that further work will lead to growth of materials other than $\mathrm{MoS}_{2}$, and production of uniform, large-area sheets of TMDCs with controllable layer number.

Chemical preparation of $\mathrm{MoS}_{2}$ (ref. 76) and $\mathrm{MoSe}_{2}$ (ref. 77) have also been demonstrated using hydrothermal synthesis (that is, growth of single crystals from an aqueous solution in an autoclave at high temperature and pressure). More recently, Matte et al. ${ }^{78,79}$ 
a

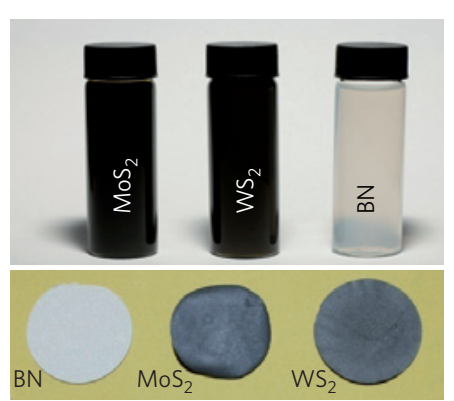

b

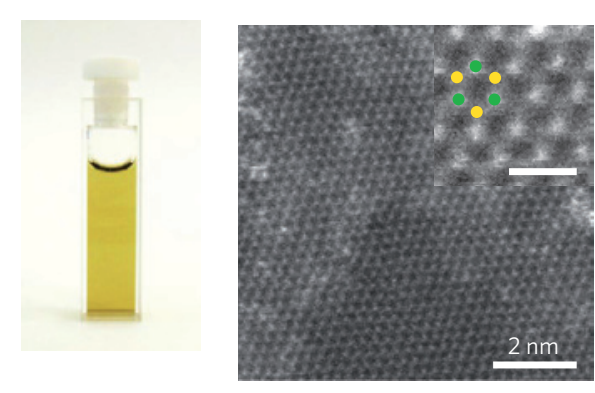

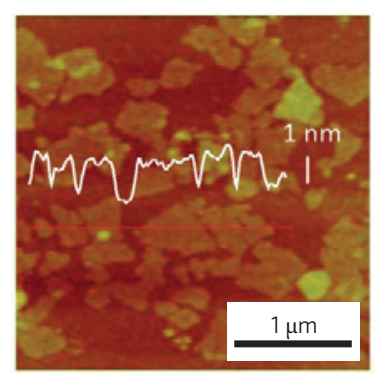

d
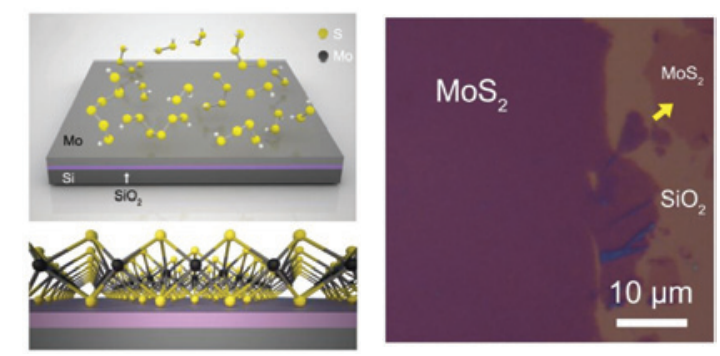

e
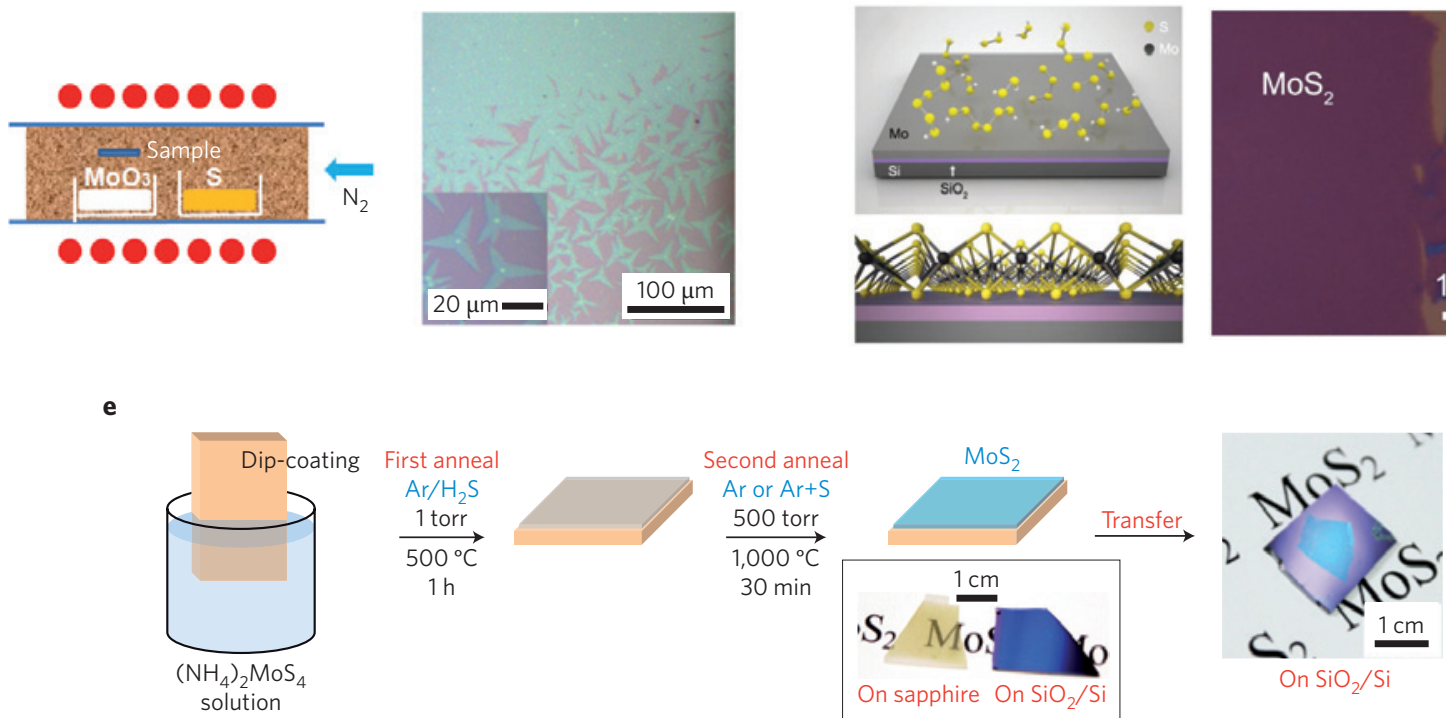

Figure 2 | Methods for synthesizing TMDC layers. a, Stable suspensions of layered materials from liquid-phase exfoliation in solvents (top), and thin films derived from vacuum filtration of these suspensions (bottom). b. Suspension of lithium-intercalated and exfoliated MoS $\mathrm{S}_{2}$ in water (left). High-angle annular dark-field scanning transmission electron microscopy (HAADF-STEM) image of $\mathrm{MoS}_{2}$ (middle) from the suspension in the left panel and atomic force microscopy (AFM) image of flakes of $\mathrm{MoS}_{2}$ deposited on $\mathrm{SiO}_{2}$ (right). The white line is a height profile taken at the position of the red line. The inset in the middle panel is a magnified view of the HAADF-STEM image, showing a honeycomb arrangement of MoS $_{2}$. Green and yellow dots represent Mo and $\mathrm{S}$, respectively. Scale bar, $0.5 \mathrm{~nm}$. c, Schematic of $\mathrm{CVD}$ of $\mathrm{MoS}_{2}$ from solid $\mathrm{S}$ and $\mathrm{MoO}_{3}$ precursors (left) and resulting $\mathrm{MoS}_{2}$ films on $\mathrm{SiO}_{2}$ (right). The red dots indicate the heating elements in the furnace. In this optical microscopy image, the lighter regions are $\mathrm{MoS}_{2}$ and the darker regions are $\mathrm{SiO}_{2}$. d, $\mathrm{CVD}$ growth of $\mathrm{MoS}_{2}$ from a solid layer of $\mathrm{Mo}_{\mathrm{O}}$ on $\mathrm{SiO}_{2}$ exposed to $\mathrm{S}$ vapour (top left), resulting in $\mathrm{MoS}_{2}$ layers that are visible in optical microscopy (right). Bottom left: side-view schematic of an $\mathrm{MoS}_{2}$ layer on the $\mathrm{Si} / \mathrm{SiO}_{2}$ substrate. e, $\mathrm{CVD}$ growth of $\mathrm{MoS}_{2}$ from a dip-coated precursor on the substrate and growth in the presence of Ar gas and S vapour. Figure reproduced with permission from: a, ref. 43, (c) 2011 AAAS; b, ref. 49, (c) 2011 ACS; c, ref. 71 , (c) 2012 Wiley; d, ref. 72, (c) 2012 Wiley; e, ref. 73, (c) 2012 ACS.

have described a number of methods to synthesize $\mathrm{WS}_{2}, \mathrm{MoS}_{2}, \mathrm{WSe}_{2}$ and $\mathrm{MoSe}_{2}$, including the reaction of molybdic or tungstic acid with either thiourea or selenourea at elevated temperatures to give the corresponding layered TMDC material ${ }^{78,79}$. Such methods give reasonably good-quality material with typical flake sizes of hundreds of nanometres to a few micrometres, although the flake thickness is not conclusively shown to be monolayers.

\section{Electronic structure}

Many TMDCs have band structures that are similar in their general features, as shown by first principles and tight-binding approximations $\mathrm{s}^{250-85}$ and measured using a variety of spectroscopic tools ${ }^{31,32,86-89}$. In general, $\mathrm{MoX}_{2}$ and $\mathrm{WX}_{2}$ compounds are semiconducting whereas $\mathrm{NbX}_{2}$ and $\mathrm{TaX}_{2}$ are metallic ${ }^{25,80-84}$. The band structures of bulk and monolayer $\mathrm{MoS}_{2}$ and $\mathrm{WS}_{2}$ calculated from first principles are shown in Fig. 3a,b (ref. 25). At the $\Gamma$-point, the bandgap transition is indirect for the bulk material, but gradually shifts to be direct for the monolayer ${ }^{24,73-77}$. The direct excitonic transitions at the K-point remain relatively unchanged with layer number ${ }^{32}$.
The change in the band structure with layer number is due to quantum confinement and the resulting change in hybridization between $p_{z}$ orbitals on $\mathrm{S}$ atoms and $d$ orbitals on Mo atoms ${ }^{31,32,81}$. The electronic distributions are also spatially correlated to the atomic structure ${ }^{32}$. For $\mathrm{MoS}_{2}$, density functional theory (DFT) calculations show that the conduction-band states at the K-point are mainly due to localized $d$ orbitals on the Mo atoms, located in the middle of the S-Mo-S layer sandwiches and relatively unaffected by interlayer coupling. However, the states near the $\Gamma$-point are due to combinations of the antibonding $p_{z}$-orbitals on the $\mathrm{S}$ atoms and the $d$ orbitals on Mo atoms, and have a strong interlayer coupling effect ${ }^{32}$. Therefore, as the layer numbers change, the direct excitonic states near the K-point are relatively unchanged, but the transition at the $\Gamma$-point shift significantly from an indirect one to a larger, direct one. All $\mathrm{MoX}_{2}$ and $\mathrm{WX}_{2}$ compounds are expected to undergo a similar indirect- to direct-bandgap transformation with decreasing layer numbers, covering the bandgap energy range 1.1-1.9 eV (refs 25,81-84,90). The bulk and monolayer bandgaps for several TMDCs are summarized in Table 1. 
a

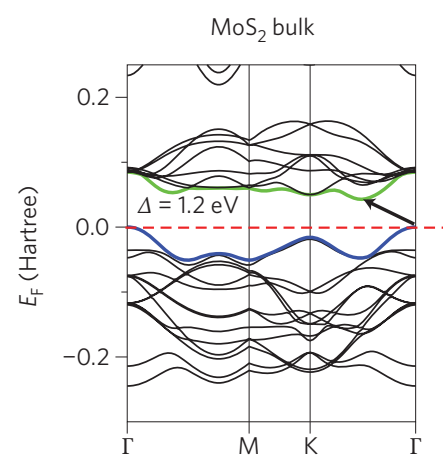

$\mathrm{MoS}_{2}$ monolayer

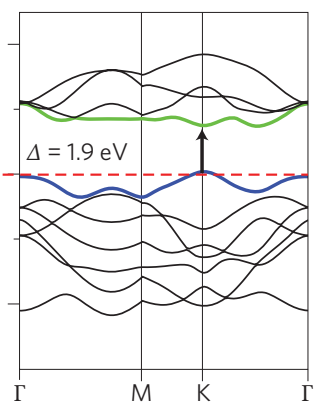

b

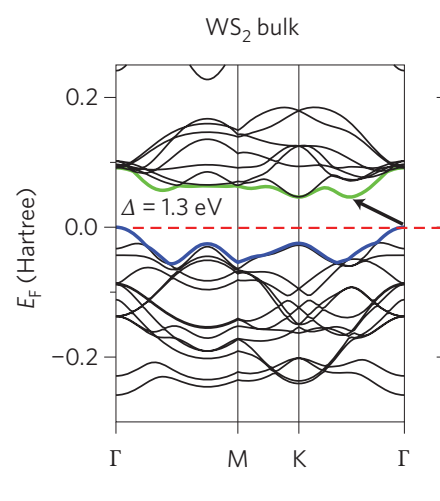

$\mathrm{WS}_{2}$ monolayer

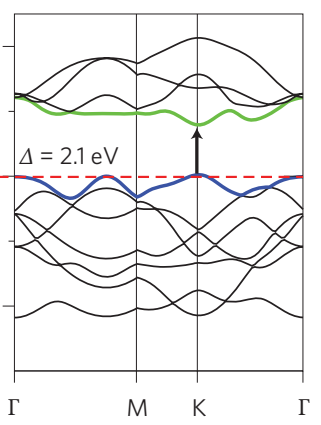

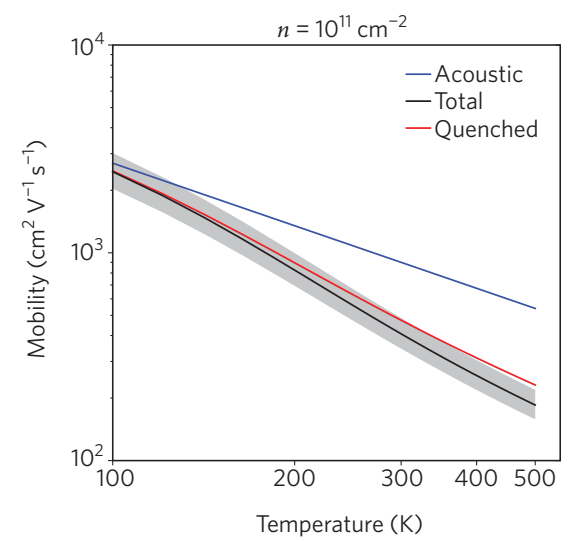

d

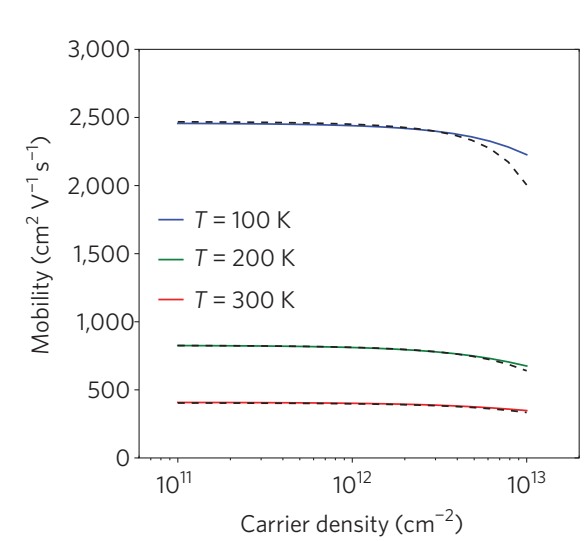

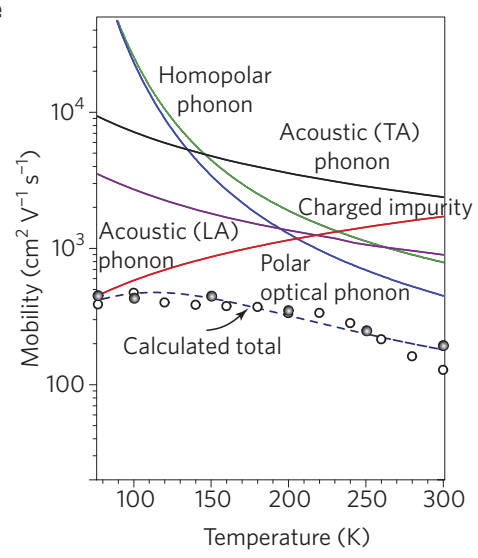

Figure 3 | Electronic properties and transport in TMDCs. a,b, Band structures calculated from first-principles density functional theory (DFT) for bulk and monolayer $\operatorname{MoS}_{2}(\mathbf{a})$ and $\mathrm{WS}_{2}(\mathbf{b})$. The horizontal dashed lines indicate the Fermi level. The arrows indicate the fundamental bandgap (direct or indirect). The top of the valence band (blue) and bottom of the conduction band (green) are highlighted. c,d, Carrier mobility in monolayer MoS ${ }_{2}$ as a function of temperature (c) and carrier density (d), calculated from first-principles DFT for the electronic band structure, phonon dispersion and electron-phonon interactions. In panel c, the grey band shows the uncertainty in calculated mobility values due to a $10 \%$ uncertainty in computed deformation potentials associated with phonons. e, Calculated and measured carrier mobility in multilayer $\mathrm{MoS}_{2}$ as a function of temperature, showing the scattering contributions from charged impurities (red line), homopolar out-of-plane phonons (green lines) and polar-optical phonons (blue line), as well as the total mobility due to the combined effects (dashed line). Figure reproduced with permission from: a,b, ref. 25, (c) 2011 APS; c,d, ref. 104, (c) 2012 APS; e, ref. 110, (c) 2012 NPG.

The bandgap in most semiconducting TMDCs, whether in bulk or monolayer, are comparable to the $1.1-\mathrm{eV}$ bandgap in silicon, as listed in Table 1, making them suitable for use as digital transistors ${ }^{91}$. The electronic band structure also determines photophysical properties. The simplified band diagram in Fig. $5 \mathrm{~d}$ shows the excitonic transitions $\mathrm{A}$ and $\mathrm{B}$, and the direct and indirect bandgaps at $E_{\mathrm{g}}$ and $E_{\mathrm{g}}{ }^{\prime}($ ref. 31).

The transition to a direct bandgap in the monolayer form has important implications for photonics, optoelectronics and sensing, as will be discussed in more detail below.

\section{Electrical transport and devices}

Materials for electronics. One of the most important applications of semiconductors is for transistors in digital electronics. In the past few decades, progress in the digital electronics industry has been driven by scaling transistors to ever-smaller dimensions. Currently, state-of-the-art processors have silicon-based metal-oxide-semiconductor field-effect transistors (MOSFETs) with feature lengths of $22 \mathrm{~nm}$ (ref. 92). Subsequent reductions in scale will soon approach limits due to statistical and quantum effects and difficulty with heat dissipation ${ }^{92}$, motivating the search for new device concepts and materials. In particular, 2D semiconductor materials are attractive for processability and lack of short-channel effects that can hinder device performance ${ }^{91}$.
In the basic FET structure, which has been adapted to $2 \mathrm{D}$ TMDCs $^{11,34}$, a semiconducting channel region is connected to the source and drain electrodes, and separated by a dielectric layer from a gate electrode ${ }^{93}$. The current flowing between the source and drain electrodes is controlled by the gate electrode modulating the conductivity of the channel. Silicon is the primary material that meets the industrial requirements for performance and manufacturability in digital logic for computing, although other semiconductors such as $\mathrm{SiC}, \mathrm{GaN}, \mathrm{Ge}$ and GaAs have more specialized applications such as light-emitting diodes (LEDs), high-power electronics, high-temperature electronics, radiofrequency electronics and photovoltaics ${ }^{94}$. Other nanomaterials that are being explored and evaluated include carbon nanotubes ${ }^{95,96}$, graphene ${ }^{68,70,95}$ and semiconductor nanowires ${ }^{97}$.

For digital logic transistors, desirable properties are high chargecarrier mobilities for fast operation, a high on/off ratio (that is, the ratio of on-state to off-state conductance) for effective switching, and high conductivity (that is, the product of charge density and mobility) and low off-state conductance for low power consumption during operation. In most semiconductors, doping can be used to increase the charge density, but can also lead to decreased mobility owing to scattering ${ }^{92,97,98}$. For digital logic, on/off ratios of $10^{4}-10^{7}$ are generally required for use as switches ${ }^{91,95}$. Much interest in graphene has centred on electronic device applications because it is two-dimensional, 
it has exceptionally high carrier mobilities, and an external gate voltage can readily modulate its current flow. ${ }^{91}$ Graphene has been used in high-frequency radiofrequency analog transistors with cutoff frequencies reaching hundreds of gigahertz, taking advantage of the high carrier mobilities and high transconductances ${ }^{68-70,91}$. But the lack of bandgap in graphene means that it cannot achieve a low offstate current, limiting its use as a digital logic transistor. There is a clear need for new nanoelectronic materials with a sizable bandgap to support high on/off ratios while maintaining high carrier mobilities and scalability to ever-smaller dimensions.

Flexibility and transparency are also desirable characteristics for next-generation electronics. Researchers are now turning to TMDCs as ultrathin materials with tunable bandgaps that can be made into FETs with high on/off ratios ${ }^{34,98}$. Two-dimensional semiconductors such as $\mathrm{MoS}_{2}$ and others offer an important advantage when compared with classical 3D electronic materials: their subnanometre thickness. Coupled with a bandgap typically in the $1-2-\mathrm{eV}$ range which can result in high on/off ratios, their extreme thinness allows more efficient control over switching ${ }^{99}$ and can help to reduce short-channel effects and power dissipation, the main limiting factors to transistor miniaturization.

Transport and scattering mechanisms. In 2D TMDC layers, transport and scattering of the carriers are confined to the plane of the material. The mobility of carriers is affected by the following main scattering mechanisms ${ }^{100,101}$ : (i) acoustic and optical phonon scattering; (ii) Coulomb scattering at charged impurities; (iii) surface interface phonon scattering; and (iv) roughness scattering. The degree to which these scattering mechanisms affect the carrier mobility is also influenced by layer thickness, carrier density, temperature, effective carrier mass, electronic band structure and phonon band structure. Many of these scattering mechanisms are also seen in other semiconductors and in graphene ${ }^{102,103}$.

Carrier mobility is increasingly affected by phonon scattering with increasing temperature ${ }^{104}$. In 2D TMDCs, which have partially ionic bonds between the metal and chalcogen atoms, crystal deformation leads to polarization fields that can interact with and scatter electrons. Figure $3 c$ shows the temperature dependence of electron mobility in single-layer $\mathrm{MoS}_{2}$ calculated from first principles by Kaasbjerg et al. ${ }^{104}$. The mobility due to acoustic phonon scattering alone is shown along with the total effect of acoustic and optical phonon scattering. At low temperatures $(T<100 \mathrm{~K})$, the acoustic component dominates, but at higher temperatures the optical component dominates. The effect of quenching the outof-plane homopolar mode, as would occur for top-gated devices, contributes only slightly to increasing the mobility (Fig. 3c). The room-temperature mobility is limited to $\sim 410 \mathrm{~cm}^{2} \mathrm{~V}^{-1} \mathrm{~s}^{-1}$, primarily owing to optical phonons ${ }^{104}$.

Coulomb scattering in 2D TMDCs is caused by random charged impurities located within the 2D TMDC layer or on its surfaces, and is the dominant scattering effect at low temperatures, as it is for graphene $\mathrm{e}^{105}$. Engineering the dielectric environment can enhance mobilities $^{106}$, as has been demonstrated for graphene $e^{107,108}$ and for $\mathrm{MoS}_{2}$ (ref. 34). This Coulomb effect also generally limits the mobility of single-layer graphene to values around $10,000 \mathrm{~cm}^{2} \mathrm{~V}^{-1} \mathrm{~s}^{-1}$ when it is placed on mildly dielectric materials such as $\mathrm{SiO}_{2}$ (ref. 109). The carrier concentration and bandgap can be tuned by adding ionic impurities, but the mobility is also decreased through scattering, so the choice of doping level in a particular device can strongly influence its performance. The effect of carrier concentration and temperature on $\mathrm{MoS}_{2}$ mobility is shown in Fig. 3d. The combined effect of phonons and charged impurities on the mobility of multilayer $\mathrm{MoS}_{2}$ is shown in Fig. 3e, and shows a similar general trend to that of monolayer $\mathrm{MoS}_{2}$ (ref. 110).

By considering the mean free path of carriers, the impurity concentration required to make impurity scattering dominant over phonon scattering is calculated to be $\sim 5 \times 10^{11} \mathrm{~cm}^{-2}$, which corresponds to heavy doping ${ }^{104}$. These results show that the improvement in room-temperature mobility from $\sim 0.5-3 \mathrm{~cm}^{2} \mathrm{~V}^{-1} \mathrm{~s}^{-1}$ in earlier reports ${ }^{1,11}$ of $\mathrm{MoS}_{2}$ transistors to $\sim 200 \mathrm{~cm}^{2} \mathrm{~V}^{-1} \mathrm{~s}^{-1}$ more recently ${ }^{34}$ is primarily due to the dielectric screening of Coulomb scattering on charged impurities by using gate dielectric materials with a high dielectric constant. Further experiments are needed to clarify the relative roles of impurity and phonon scattering.

The effect of surface phonon scattering and roughness scattering can be very important in extremely thin $2 \mathrm{D}$ materials. In GaAs-based quantum wells, interfacial roughness scattering can dominate $^{111}$. In the case of graphene, Coulomb scattering is dominant over short-range scattering and surface roughness in the form of ripples ${ }^{103}$. Graphene placed on a dielectric material such as $\mathrm{SiO}_{2}$, however, experiences scattering due to surface polar phonons in the $\mathrm{SiO}_{2}$ (ref. 102), and in freely suspended graphene $e^{105,109}$ the primary scattering is due to out-of-plane flexural phonons ${ }^{112}$. Additionally, freestanding $\mathrm{MoS}_{2}$ has been shown to have similar ripples ${ }^{113}$ to those in graphene, which may also contribute to scattering and mobility reduction. The phonon-limited roomtemperature mobility calculated by Kaasbjerg et al. ${ }^{104}$ for $\mathrm{MoS}_{2}$ is $\sim 410 \mathrm{~cm}^{2} \mathrm{~V}^{-1} \mathrm{~s}^{-1}$, and similar values are expected for other singlelayer TMDCs.

TMDC transistors. Semiconducting 2D TMDCs have unique features that make them attractive as a channel material in FETs such as the lack of dangling bonds, structural stability and mobility comparable to Si (ref. 114). One of the earliest uses of TMDCs in FETs was reported in 2004, where $\mathrm{WSe}_{2}$ crystals showed mobility comparable to the best single-crystal Si FETs (up to $500 \mathrm{~cm}^{2} \mathrm{~V}^{-1} \mathrm{~s}^{-1}$ for p-type conductivity at room temperature), ambipolar behaviour and a $10^{4} \mathrm{on} / \mathrm{off}$ ratio at a temperature of $60 \mathrm{~K}$ (ref. 115). This result was soon followed by devices based on thin layers of $\mathrm{MoS}_{2}$ with a back-gated configuration, resulting in mobility values in the range $0.1-10 \mathrm{~cm}^{2} \mathrm{~V}^{-1} \mathrm{~s}^{-1}$ (refs 1,11).

The first implementation of a top-gated transistor based on monolayer $\mathrm{MoS}_{2}$ was reported by Kis and co-workers ${ }^{34}$, as shown in Fig. 4a. This device showed excellent on/off current ratio $\left(\sim 10^{8}\right)$, n-type conduction, room-temperature mobility of $>200 \mathrm{~cm}^{2} \mathrm{~V}^{-1} \mathrm{~s}^{-1}$ and subthreshold swing of $74 \mathrm{mV}$ per decade ${ }^{34}$. Current-voltage curves for this FET are shown in Fig. 4b. The top-gated geometry allows for a reduction in the voltage necessary to switch the device while allowing the integration of multiple devices on the same substrate. The high- $k$ dielectric used in this device, $\mathrm{HfO}_{2}$, also gave the additional benefit of improving the mobility of monolayer $\mathrm{MoS}_{2}$ owing to dielectric engineering as discussed earlier ${ }^{106,107,116,117}$. Topgating with a high- $k$ dielectric was also used in a p-type FET with an active channel made of a monolayer flake of $\mathrm{WSe}_{2}$, which exhibited room-temperature performance of $\sim 250 \mathrm{~cm}^{2} \mathrm{~V}^{-1} \mathrm{~s}^{-1}$ hole mobility, $\sim 60 \mathrm{mV}$ per decade subthreshold swing and $10^{6}$ on/off ratio ${ }^{118}$. Thin-film transistors made of $\mathrm{MoS}_{2}$ from liquid exfoliation also have similar electrical performance ${ }^{119}$, suggesting possibilities for flexible, transparent, 2D electronic applications. The development of CVD synthesis methods for obtaining large areas of $\mathrm{MoS}_{2}$, as described earlier, is also important for wafer-scale fabrication of devices.

Theoretical simulations of single-layer $\mathrm{MoS}_{2}$ transistor performance $e^{82,98}$ have quantified the expected resilience of $\mathrm{MoS}_{2}$ to shortchannel effects due to its atomic-scale thickness. These calculations show that top-gated $\mathrm{MoS}_{2}$ transistors with gate lengths of $15 \mathrm{~nm}$ could operate in the ballistic regime with on-current as high as

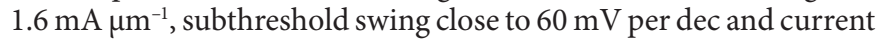
on/off ratio of $10^{10}$. Simulated current-voltage curves for a singlelayer $\mathrm{MoS}_{2}$ transistor at different operating conditions are shown in Fig. 4d (ref. 98). Although $\mathrm{MoS}_{2}$ will not compete with conventional III-V transistors on mobility values alone, its attractive electrical 
a

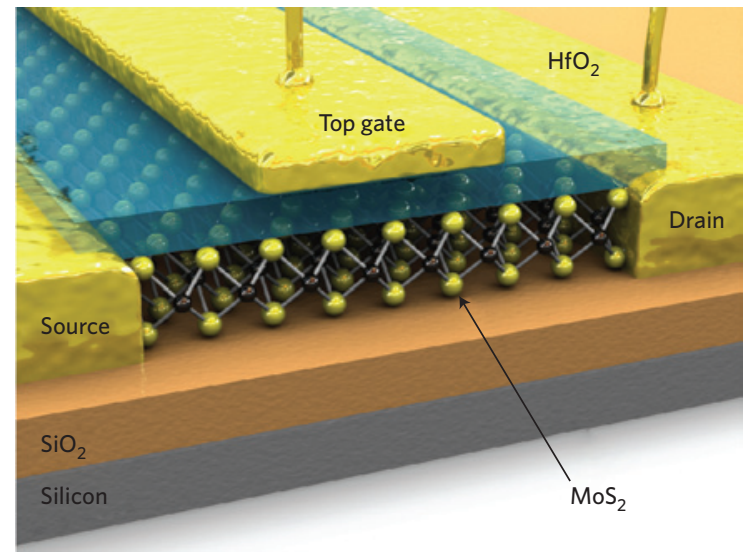

c

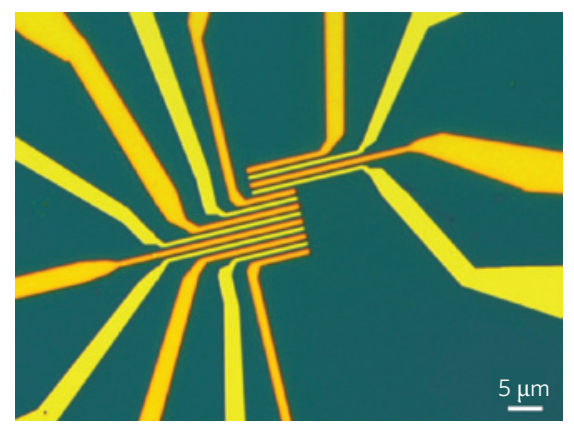

b

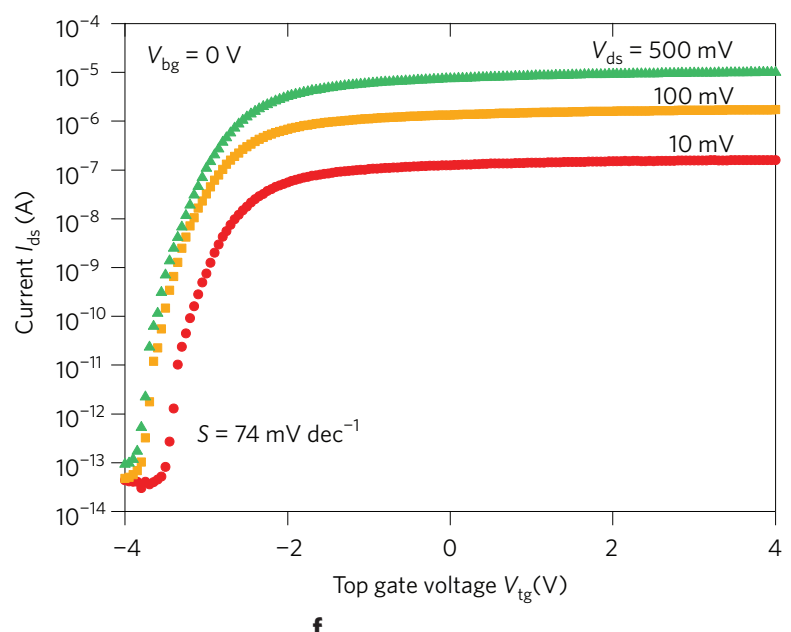

f

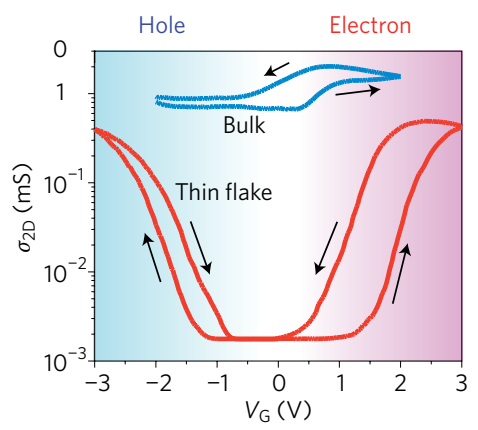

d



g

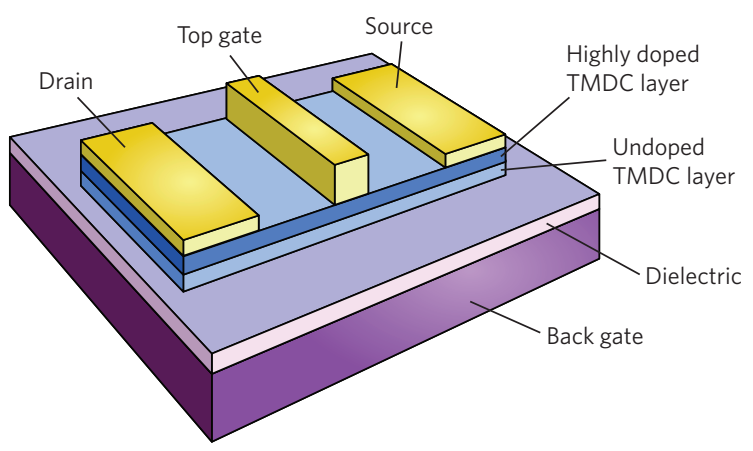

Figure 4 | Electronic devices from thin flakes of $\mathrm{MoS}_{2}$. $\mathbf{a}$, Schematic illustration of $\mathrm{HfO}_{2}$-top-gated monolayer MoS $\mathrm{FET}$ device. b, Source-drain current $\left(I_{\mathrm{ds}}\right)$ versus top gate voltage $\left(V_{\mathrm{tg}}\right)$ curve recorded from the top-gated device in a for a bias voltage ranging from $10 \mathrm{mV}$ to $500 \mathrm{mV}$. Measurements are performed at room temperature with the back gate grounded. Top gate width, $4 \mathrm{~mm}$; top gate length, $500 \mathrm{~nm}$. The device can be completely turned off by changing the top gate bias from -2 to $-4 \mathrm{~V}$. For $V_{\mathrm{ds}}=10 \mathrm{mV}$, the $I_{\text {on }} / I_{\text {off }}$ ratio is $>1 \times 10^{6}$. For $V_{\mathrm{ds}}=500 \mathrm{mV}$, the $I_{\text {on }} / I_{\text {off }}$ ratio is $>1 \times 10^{8}$ in the measured range while the subthreshold swing $S=74 \mathrm{mV} \mathrm{dec}^{-1}$. c, Integrated circuit based on single-layer $\mathrm{MoS}_{2}$. d, Simulated device characteristics for a monolayer $\mathrm{MoS}_{2}$ FET device with 2.8-nm-thick $\mathrm{HfO}_{2}$ top-gate oxide, 15 -nm gate length, and power supply voltage $0.5 \mathrm{~V}$. The source-drain current $\left(I_{\mathrm{D}}\right)$ is plotted against gate voltage $\left(V_{G}\right)$ for 0.05 and $0.5 \mathrm{~V}$ drain voltage $\left(V_{D}\right)$ on linear (right axis) and logarithmic (left axis) scales. e, Schematic of electric double-layer transistor (EDLT, a FET gated by ionic liquids). $V_{D S}$ is the source-drain voltage and $V_{G}$ is the gate voltage. $\mathbf{f}$, Conductivity as a function of top gate voltage for both bulk and thin-flake $M_{2} S_{2}$ EDLT devices. Thin-flake devices show on/off ratios $>10^{2}$ for both electron and hole transport. $\mathbf{g}$, Proposed TMDC-based high-electron-mobility transistor (HEMT) device with top-gated Schottky contact and TMDC layers with different doping levels. Figure reproduced with permission from: a,b, ref. 34, (c) 2011 NPG; d, ref. 98, (c) 2011 ACS; e,f, ref. 41, (c) 2012 ACS.

performance characteristics, relatively high Earth abundance and high degree of electrostatic control could make $\mathrm{MoS}_{2}$ a viable candidate for low-power electronics ${ }^{98}$.

Radisavljevic et al. ${ }^{35}$ recently demonstrated that they could build functional electronic circuits based on multiple 2D TMDC transistors capable of performing digital logic operations. Up to six independently switchable transistors were fabricated on the same piece of monolayer $\mathrm{MoS}_{2}$ by lithographically patterning multiple sets of electrodes (Fig. 4c) ${ }^{35}$. An integrated circuit composed of two transistors fabricated on a single flake of $\mathrm{MoS}_{2}$ was operated as a logical inverter, which converts a logical 0 into a logical 1, and as a logical NOR gate ${ }^{35}$, which is one of the universal gates that can be built 
in combinations to form all other logic operations ${ }^{120}$. Wang et al. also recently demonstrated complex integrated circuits built on bilayer $\mathrm{MoS}_{2}$, including an inverter, logical NAND gate, static random access memory and five-stage ring oscillator ${ }^{121}$.

Ambipolar transport was demonstrated in a thin (10-nm-thick) $\mathrm{MoS}_{2}$ electric double-layer transistor (EDTL) using an ionic liquid as the gate (Fig. $4 \mathrm{e}$ ) to reach extremely high carrier concentrations of $1 \times 10^{14} \mathrm{~cm}^{2}$ (ref. 41). The demonstration of both $\mathrm{n}$ - and p-type transport will be useful for applications like CMOS logic and $\mathrm{p}-\mathrm{n}$ junction optoelectronics. The on/off ratio was $>200$ in the device, but is much lower than the single-layer device described above ${ }^{34}$, mainly because of the off-current passing through the bulk of the flakes ${ }^{41}$. The transfer curves (source-drain current as a function of gate voltage) for thin-flake and bulk $\mathrm{MoS}_{2}$ ambipolar devices are shown in Fig. 4f.

Future directions for TMDC electronics. One of the most promising near-term applications of semiconducting 2D TMDCs is in high-performance flexible electronics. Mechanical measurements performed on single-layer $\mathrm{MoS}_{2}$ show that it is 30 times as strong as steel and can be deformed up to $11 \%$ before breaking ${ }^{33}$. This makes $\mathrm{MoS}_{2}$ one of the strongest semiconducting materials and advantageous for flexible substrates. Flexible transistors have been demonstrated using electrochemically lithiated and exfoliated $\mathrm{MoS}_{2}$ as gas sensors ${ }^{122}$ and CVD-grown $\mathrm{MoS}_{2}$ with an ionic gel gate dielectric ${ }^{123}$. Other dichalcogenide semiconductors display mechanical properties similar to $\mathrm{MoS}_{2}$ (ref. 84). Semiconducting 2D TMDCs, combined with other 2D materials such as conducting graphene and insulating $\mathrm{BN}$, can form hybrid all-2D electronics on flexible substrates.

Another potential application is in a 2D TMDC analogue of high-electron-mobility transistors (HEMTs), which are conventionally fabricated from planar junctions of semiconductors of different bandgaps such as GaAs and AlGaAs using molecular beam evaporation techniques ${ }^{124}$. In these devices, the semiconductor with the smaller bandgap is highly doped whereas the one with the larger bandgap is left undoped, such that when the two layers are brought into contact the electrons from the doped layer move to the undoped layer and are free to move with minimal scattering from dopants. TMDCs could be adapted to this device architecture because the different TMDCs have a range of bandgaps (Table 1) and similar lattice constants ${ }^{83}$. They are also generally more Earth-abundant and processable than current materials. The proposed 2D TMDC HEMT device is shown in Fig. 4g, where a highly doped TMDC layer is interfaced with an undoped layer. The gate electrode modulating the signal through the device is generally a reverse-biased Schottky contact. Some of the limitations of TMDCs in electronic devices, however, include the relatively high effective mass of carriers and relatively low carrier mobility values ${ }^{98}$, which may restrict certain high-performance applications.

Another promising research direction is the stacking of different $2 \mathrm{D}$ layered materials together to make vertical heterostructures and hybrid devices with operating principles that are unlike conventional devices. Recently, a new device architecture called a field-effect tunnelling transistor was put forth ${ }^{125}$, in which a high switching ratio was achieved using two independently controlled graphene layers are separated by thin $\mathrm{MoS}_{2}$ or hexagonal boron nitride $(\mathrm{hBN})$ layers acting as tunnelling barriers.

\section{Optoelectronics}

Optoelectronic devices are electronic devices that can generate, detect, interact with or control light. Nanomaterials such as carbon nanotubes, semiconductor quantum dots and nanowires have been much studied for use in optoelectronic applications such as lasers, LEDs, solar cells, optical switches, photodetectors and displays ${ }^{96,126-128}$. Optoelectronic devices that are flexible and transparent are expected to become increasingly important in solar arrays, wearable electronics and transparent displays. The electronic band structures of semiconductors directly influence their ability to absorb and emit light. For semiconductors with a direct bandgap, photons with energy greater than the bandgap energy can be readily absorbed or emitted. For indirect bandgaps, an additional phonon must be absorbed or emitted to supply the difference in momentum, making the photon absorption or emission process much less efficient. Because single-layer TMDCs have primarily direct semiconducting bandgaps, they are of great interest for applications in optoelectronics, and because they are atomically thin and processable, they have great potential for flexible and transparent optoelectronics.

Optical and vibrational properties of TMDCs. The electronic band structures of TMDCs described earlier directly influence their optical properties. For $\mathrm{MoS}_{2}$, the change from indirect to direct bandgap and increase in bandgap energy is observed in changes in photoconductivity, absorption spectra and photoluminescence ${ }^{25,31,32}$. This shift results in up to a factor of $10^{4}$ increase in photoluminescence quantum yield from bulk to monolayer $\mathrm{MoS}_{2}$, with even higher quantum yield for regions of the $\mathrm{MoS}_{2}$ monolayer flake suspended over holes in the substrate (Fig. 5e) ${ }^{31}$. But the overall quantum yield of $\mathrm{MoS}_{2}$ measured so far - about $10^{-5}-10^{-6}$ for few-layer samples and up to $4 \times 10^{-3}$ for monolayer samples ${ }^{31}$ - is significantly lower than the near-unity values that would be expected for a direct-gap semiconductor. The observations of quantum yield being higher for suspended samples ${ }^{31}$ and samples placed on hBN substrates ${ }^{26}$ than on $\mathrm{SiO}_{2}$ suggest that more work is needed to understand and control the quenching mechanisms in $\mathrm{MoS}_{2}$ and other TMDCs, and to increase the quantum yield for future optoelectronic applications.

The main peak of the monolayer $\mathrm{MoS}_{2}$ photoluminescence spectrum is the direct-gap luminescence feature at $1.9 \mathrm{eV}$ (ref. 31), as seen in Fig. 5f, whereas few-layer $\mathrm{MoS}_{2}$ also has additional peaks corresponding to the indirect-gap luminescence, and direct-gap hot luminescence ${ }^{31}$. The photoconductivity of TMDCs also reflects these band-structure features, with the photocurrent of $\mathrm{MoS}_{2}$ increasing in steps with respect to photon energy corresponding to the direct and indirect gap energies ${ }^{31}$. The optical absorption spectrum of bulk $\mathrm{MoS}_{2}$ shows two main peaks corresponding to exciton bands, the so-called A and B excitons: direct-gap transitions at the K-point of the Brillouin zone between the maxima of split valence bands and the minimum of the conduction band ${ }^{31,32,129}$ (Fig. 5d). Calculations have shown that the $\mathrm{A}$ and $\mathrm{B}$ excitons correspond to the expected energies of the gap energies at the K-point ${ }^{130}$, where the band splitting is due to spin-orbit coupling in monolayer $\mathrm{MoS}_{2}$ (spin-orbit effects are described in more detail below). The calculations have also shown that the exciton binding energies are quite high owing to the decreased dielectric constants compared with the bulk, as well as the $2 \mathrm{D}$ confinement: about $0.897 \mathrm{eV}$ for monolayer $\mathrm{MoS}_{2}$ and $0.424 \mathrm{eV}$ for bilayer ${ }^{131}$. We also note that in these calculations the transition energy of the excitons is offset by the exciton binding energy, and so the energy required to create an exciton would be much lower than the bandgap, and that the optical transition energies are not equivalent to the transport bandgap energies.

The phonon dispersions of $\mathrm{MoS}_{2}$ and $\mathrm{WS}_{2}$ have been calculated $a b$ initio ${ }^{132}$, and that of $\mathrm{MoS}_{2}$ was correlated with experimental Raman spectra ${ }^{30}$. The main Raman peaks correspond to the inplane $E_{2 g}^{1}$ and $E_{1 u}$ phonon modes, and the out-of-plane $A_{1 g}$ mode (Fig. 5a). For decreasing layer thickness, the $A_{1 g}$ mode near $406 \mathrm{~cm}^{-1}$ decreases in frequency whereas the $\mathrm{E}_{2 \mathrm{~g}}^{1}$ mode near $382 \mathrm{~cm}^{-1}$ increases (Fig. 5b $)^{30}$. These peak position shifts allow layer thicknesses to be identified through Raman spectroscopy. The origins of the shifts have been identified as the influence of neighbouring layers on the effective restoring forces on atoms and the increase of dielectric screening of long-range Coulomb interactions ${ }^{132}$. 
a



d

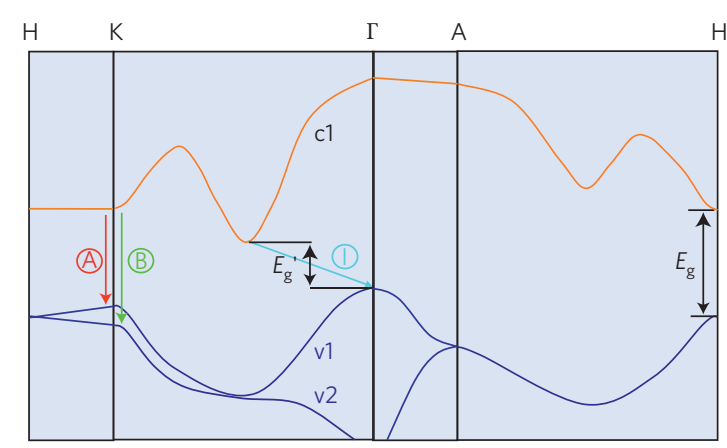

f

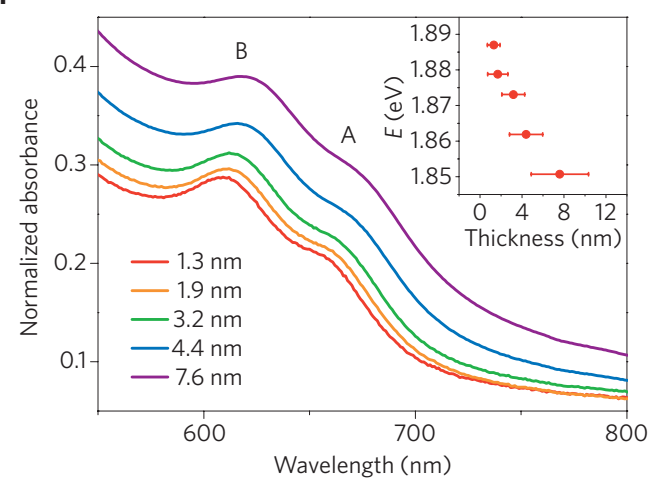

\section{(1)}

b

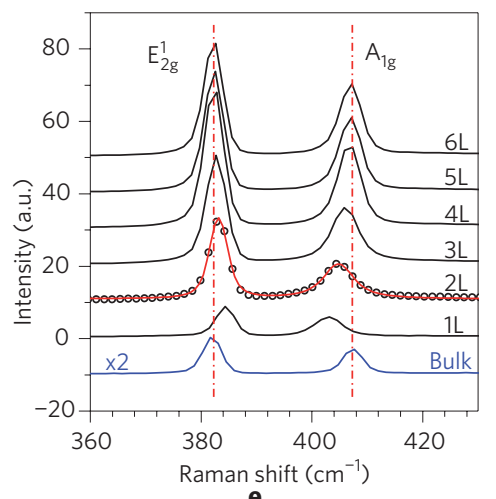

c



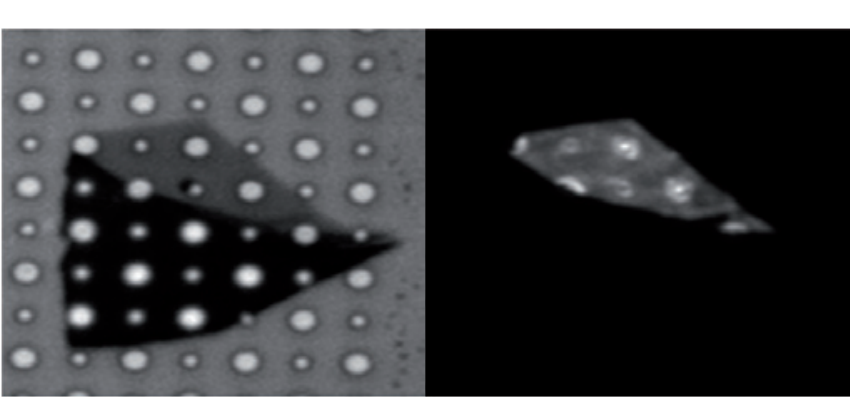

g

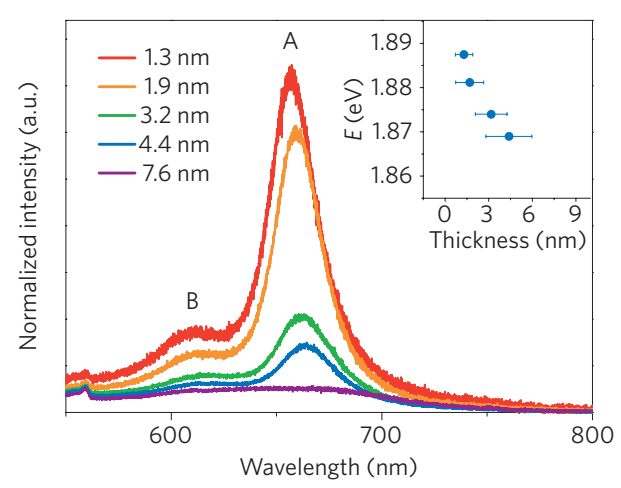

Figure 5 | Optical and vibrational properties. a, Schematic illustration of in-plane phonon modes $E_{2 g}^{1}$ and $E_{1 u}$ and the out-of-plane phonon mode $A_{1 g^{\prime}}$ for the bulk $\mathrm{MoS}_{2}$ (analogously for $\mathrm{WS}_{2}$ ). b. Thickness-dependent Raman spectra for $\mathrm{MoS}_{2}$. c, Peak position shifts for the $\mathrm{E}_{2 \mathrm{~g}}^{1}$ and $\mathrm{A}_{1 \mathrm{~g}}$ modes as a function of MoS layer thickness for the spectra in $\mathbf{b}$. d, Simplified band structure of $\mathrm{MoS}_{2}$ showing the lowest conduction band $\mathrm{c} 1$ and the highest split valence bands $\mathrm{v} 1$ and v2. $A$ and $B$ are the direct-gap transitions, and I is the indirect-gap transition. $E_{g}{ }^{\prime}$ is the indirect gap for the bulk, and $E_{g}$ is the direct gap for the monolayer. e, Optical (left) and photoluminescence images (right) of $\mathrm{MoS}_{2}$ flake on $\mathrm{SiO}_{2}$ substrate with etched holes. The large holes in the left panel are $1.5 \mu \mathrm{m}$ in diameter. The photoluminescence emission is enhanced in suspended regions over holes and in monolayer $\mathrm{MoS}_{2}$, but is not detected in the multilayer region. $\mathbf{f}, \mathbf{g}$, Absorption (f) and photoluminescence spectra $(\mathbf{g})$ of $\mathrm{MoS}_{2}$ thin films with average thicknesses ranging from 1.3 to $7.6 \mathrm{~nm}$. Insets of $\mathbf{f}$ and $\mathbf{g}$ show energy of the A exciton peak as a function of average film thickness. The peak energies were extracted from the absorption and photoluminescence spectra in the main panel, respectively. Figure reproduced with permission from: a, ref. 132, (c) 2011 APS; b,c, ref. 30, (c) 2010 ACS; d,e, ref. 31, (c) 2010 APS; f,g, ref. 49, (c) 2011 ACS.

Flexible and transparent optoelectronics. The realization of flexible and transparent optoelectronics in applications such as displays and wearable electronics will require a wide variety of transparent and flexible components such as conductors, semiconductors, optical absorbers, light emitters and dielectrics. These diverse functionalities will require integrating different classes of 2D materials with different properties. For transparent conductors, graphene's high conductivity and low broadband absorption makes it a promising flexible and earth-abundant replacement ${ }^{133-136}$ for the current leading material, indium tin oxide, which is inflexible and increasingly expensive owing to the scarcity of indium. For semiconducting components with tunable bandgaps, 2D TMDCs are a promising choice and can be formed into light-absorbing components or light-emitting devices. For 2D dielectrics, materials such as layered perovskites ${ }^{10}$ and $\mathrm{BN}$ (ref. 12) are promising.

Photovoltaics and photodetection. The relatively high Earth abundance of TMDCs and their direct bandgaps in the visible range make them attractive as the light-absorbing material in alternative thin-film solar cells $s^{137}$, including flexible photovoltaics that could coat buildings and curved structures. The workfunctions and conduction- and valence-band edges of several TMDCs are compatible with the workfunctions of commonly used electrode materials ${ }^{138-140}$. Moreover, the ability to tune the bandgap of TMDCs with various 
a

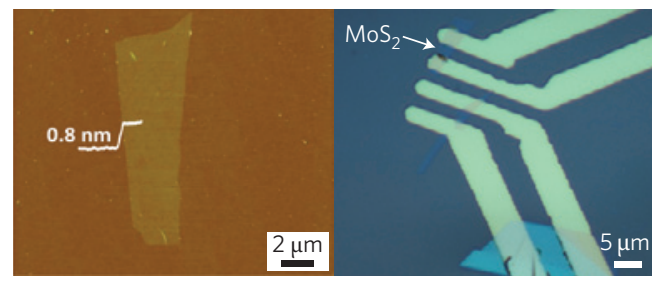

C


b

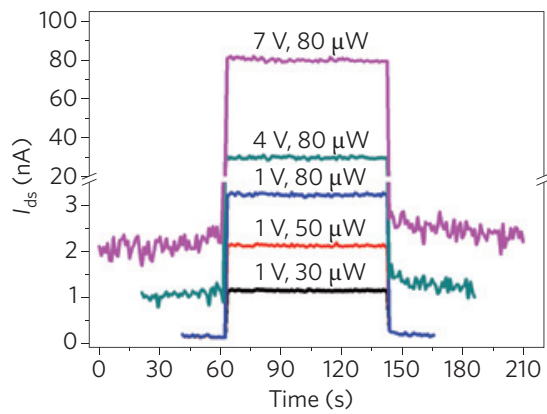

d

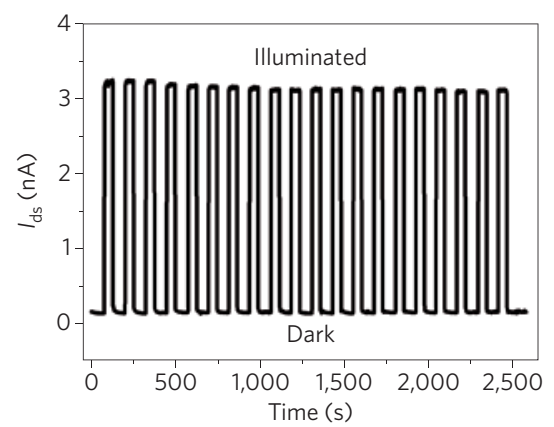

f

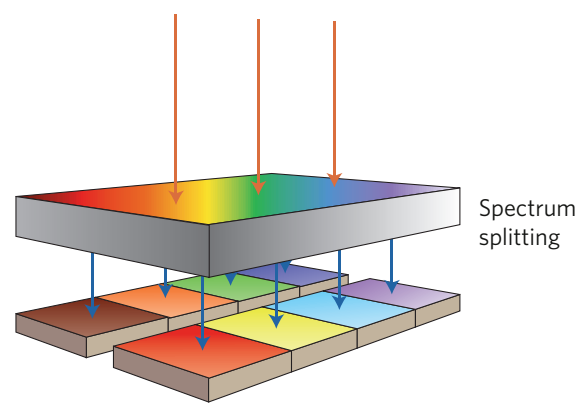

Figure 6 | Current and proposed optoelectronic devices. a, Atomic force microscopy image of MoS $_{2}$ monolayer flake (left) and optical microscopy image of this flake made into a device with metal contacts (right). The white trace in the left panel shows a height profile from AFM along the MoS $\mathrm{flake}$ edge. b, Photoswitching characteristics of single-layer $\mathrm{MoS}_{2}$ phototransistor at different optical power $\left(P_{\text {light }}\right)$ and drain voltage $\left(V_{\mathrm{ds}}\right)$. c, Photoswitching rate and $\mathbf{d}$, stability test of photoswitching behaviour of single-layer $\mathrm{MoS}_{2}$ phototransistor at $V_{\mathrm{ds}}=1 \mathrm{~V}, P_{\text {light }}=80 \mu \mathrm{W}$. e, Energy-level diagram of proposed multijunction solar cell made of stacked semiconductors of different bandgaps to absorb different wavelengths from the solar spectrum to reduce thermalization losses. The blue dashed lines represent the quasi-Fermi levels defining the open-circuit voltage, and yellow dots represent electrons in the device. f, Proposed solar-cell device with parallel structures that may be fabricated using lift-off and printing techniques for patterning the semiconductor layers and a micro- or nanophotonic spectrum-splitting layer. The various bandgaps available from the different TMDCs are promising for use in such multijunction photovoltaic devices. Figure reproduced with permission from: a-d, ref. 39, (C) 2012 ACS; e,f, ref. 149, (c) 2012 NPG.

intercalants such as metal ions and organic molecules ${ }^{141,142}$ may allow optical absorbances to be tuned in photovoltaic applications.

A variety of roles for TMDCs in photovoltaics and photodetectors has been demonstrated. Thin films of $\mathrm{MoS}_{2}$ and $\mathrm{WS}_{2}$ are photosensitive ${ }^{143}$, and a phototransistor made from a single layer of $\mathrm{MoS}_{2}$ has shown its potential as a photodetector (Fig. 6a-d) ${ }^{39}$. The photocurrent in this device depends on the incident light intensity, responds within $50 \mathrm{~ms}$ to changes in light levels and has high photoresponsivity. By using $\mathrm{MoS}_{2}$ layers of different thicknesses, photodetection of different wavelengths can be tuned. Lee et al. ${ }^{144}$ have demonstrated that single- and double-layer $\mathrm{MoS}_{2}$, with respective bandgap energies of 1.8 and $1.65 \mathrm{eV}$, are effective for detecting green light, and triple-layer $\mathrm{MoS}_{2}$ with a bandgap of $1.35 \mathrm{eV}$ is well suited for red light. A bulk heterojunction solar cell made from $\mathrm{TiO}_{2}$ nanoparticles, $\mathrm{MoS}_{2}$ atomic layer nanosheets and poly(3-hexylthiophene) (P3HT) was recently demonstrated with $1.3 \%$ photoconversion efficiency ${ }^{145}$. Similarly, electrochemical solar cells with $\mathrm{TiO}_{2}$ were sensitized with $\mathrm{WS}_{2}$, which acts as a stable, inorganic absorber material ${ }^{146,147}$. TMDCs have also been demonstrated as conductors and electron-blocking layers in polymer LEDs ${ }^{53,148}$.

In Fig. 6e,f, two proposed devices that incorporate materials with different bandgaps are shown: multijunction solar cells like these would allow photons of different energies in the full solar spectrum to be efficiently absorbed, reducing losses due to thermalization $^{149}$. These structures could potentially be constructed using different TMDCs with varying bandgaps, which range from the visible to the near-infrared, as summarized in Table 1 . The layer-dependent photodetector ${ }^{144}$ discussed earlier demonstrates how light of different wavelengths can be preferentially absorbed by tuning the bandgap.

Light emission. Electroluminescence and photoluminescence are two important categories. In electroluminescence, photons are emitted in response to electrical stimulus; this mode is useful in 
optoelectronic devices such as LEDs and diode lasers. In photoluminescence, the material absorbs a photon and then re-radiates a photon, sometimes at a different energy. In direct-bandgap semiconductors, the radiative recombination of electrons and holes produces photons, and occurs much more efficiently than in indirect-bandgap semiconductors. The direct bandgaps of monolayer semiconducting TMDCs make them ideal candidates for the active light-emitting layer in future flexible optoelectronics, unlike graphene, which lacks a bandgap and requires chemical treatments to induce local bandgaps that photoluminesce ${ }^{150,151}$. Examples of electroluminescence in TMDCs include $\mathrm{MoS}_{2}$ emitting light by electrical excitation through Au nano-contacts ${ }^{152}$, and electroluminescence from $\mathrm{SnS}_{2}$ exfoliated from lithium intercalation and incorporated into a composite polymer matrix ${ }^{56}$. Photoluminescence is seen in monolayer $\mathrm{MoS}_{2}$, which has a direct bandgap, and the quantum yield of the monolayer photoluminescence is much higher than for bilayer and bulk $\mathrm{MoS}_{2}$ (refs 32, 49). As mentioned earlier, however, the photoluminescent quantum yield measured so far in $\mathrm{MoS}_{2}$ is much lower than would be expected for a direct-gap semiconductor, and much work will be required to understand the photoluminescence emission and quenching processes before many feasible optoelectronic devices can be made.

\section{Spin, orbit and valley interactions}

Electronic and spintronic devices use electronic charge and spin, respectively, to carry signals. The valley index is another property of charge carriers that can be exploited, and it refers to the confinement of electrons or holes in distinct conduction-band minima or valence-band maxima at the same energies but different positions in momentum space, leading to potential 'valleytronic' devices ${ }^{153}$. Materials that have strong spin splitting, which can be due to various effects that push the system out of equilibrium or due to symmetry breaking, allow spin-polarized carrier populations to be maintained, and are needed for spintronic devices.

In the group-IV semiconducting dichalcogenides $\left(\mathrm{MoS}_{2}, \mathrm{MoSe}_{2}\right.$, $\mathrm{WS}_{2}$ and $\mathrm{WSe}_{2}$ ), a unique set of conditions gives rise to both strong spin-orbit-induced electronic band splitting ${ }^{154}$ and spin-valley coupling ${ }^{153}$. Monolayer TMDCs such as $\mathrm{MoS}_{2}$ lack inversion symmetry, as seen in the structural diagrams in Fig. 1e (ref. 153), unlike graphene or bilayer $\mathrm{MoS}_{2}$, which are centrosymmetric. The lack of inversion symmetry, confinement of electron motion in plane and high mass of the elements in the $\mathrm{MX}_{2}$ materials lead to a very strong spin-orbit splitting, with the valence-band splittings ranging between 0.15 and $0.45 \mathrm{eV}$ (ref. 154). This is in contrast to graphene, which has very weak spin-orbit interaction primarily owing to the low mass of carbon. The strong spin splitting in the $\mathrm{MX}_{2}$ materials makes them promising candidates for spintronic devices.

The lack of inversion symmetry along with strong spin-orbit coupling also leads to the coupling of spin and valley physics ${ }^{153}$. Experimental evidence of valley confinement has been seen in monolayer $\mathrm{MoS}_{2}$, where the carrier populations in distinct valleys can be controlled by optically exciting the samples with circularly polarized light, as recently demonstrated by three independent groups $^{26-28}$. This development may be the first step towards a new field of valleytronic devices ${ }^{155,156}$. These spin, orbit and valley properties are quite distinctive in TMDCs and may lead to as yet unforeseen applications.

\section{Molecular sensing applications}

The electronic, optoelectronic and chemical properties of TMDCs suggest opportunities in molecular sensing applications. As 2D materials, their high surface-to-volume ratio makes them particularly sensitive to changes in their surroundings. On exposure to gases and vapours, there can be changes such as charge transfer and doping, intercalation, and shifts in permittivity and lattice vibrations. For example, gas sensors from a graphene-based FET can perform with high sensitivity and low noise. The detection mechanism is due to adsorbed molecules transferring charge to the graphene sheet and changing its resistivity ${ }^{157-159}$.

Changes to the electronic properties of TMDCs caused by the presence of adsorbates can be detected electrically by incorporating the TMDCs into transistor devices and measuring the current-voltage behaviour, or optically by changes in the photoluminescence, absorbance or Raman spectra. For example, intercalation with $\mathrm{Li}^{+}$ ions causes an upshift in the $\mathrm{MoS}_{2}$ Raman $\mathrm{A}_{1 \mathrm{~g}}$ and $\mathrm{E}_{2 \mathrm{~g}}^{1}$ peak positions and a decrease in peak intensities, probably owing to strain introduced by the $\mathrm{Li}^{+}$entering interstitial sites ${ }^{74,160}$. The photoluminescence of monolayer $\mathrm{MoS}_{2}$ also suggests applications in biosensing, where stable fluorescent markers are of importance for imaging and fluorometric assays.

Transistors made from single- and few-layer $\mathrm{MoS}_{2}$ sheets have been demonstrated to be sensitive detectors for $\mathrm{NO}$ gas $^{161}$. The detection mechanism is probably due to p-doping induced by the adsorbed NO, changing the resistivity of the intrinsically $n$-doped $\mathrm{MoS}_{2}$. Similarly, flexible thin-film transistors with active regions composed of $\mathrm{MoS}_{2}$ generated from the electrochemical lithiation and exfoliation technique ${ }^{50}$ have been demonstrated as sensitive $\mathrm{NO}_{2}$ gas detectors ${ }^{122}$. Thin-film humidity sensors made from liquid-phase exfoliated $\mathrm{VS}_{2}$ nanosheets have been incorporated into a sensor array that can detect the moisture from fingers ${ }^{40}$. The conductivity in these sensors is modulated by the presence of water molecules, which inhibit conduction along $\mathrm{V}$ atoms at flake edges ${ }^{40}$. Transistors made from $\mathrm{MoS}_{2}$ also exhibit humidity-dependent hysteresis in their current-voltage behaviour, probably owing to water molecules easily adsorbing onto the hydrophilic $\mathrm{MoS}_{2}$ surface and being polarized by the applied gate voltage ${ }^{162}$. Finally, $\mathrm{MoS}_{2}$ flakes incorporated into a glassy carbon electrode in an electrochemical cell can be electrochemically reduced, and this reduced material has been shown to have electrochemical sensitivity toward glucose and biomolecules such as dopamine $e^{163}$.

\section{D materials for the future}

Layered TMDC materials have been known and studied for decades, but their properties as atomically thin, $2 \mathrm{D}$ forms are a relatively new and exciting area for nanotechnology, with many promising applications in nanoelectronics and optoelectronics. The large bandgaps seen in several members of the TMDC family make them attractive channel materials in logic transistors, and the direct bandgaps in several single-layer TMDCs open up many prospects in optoelectronics. Two-dimensional $\mathrm{MoS}_{2}$ has been used to demonstrate transistors with high on/off ratios and integrated circuits with logic operation, as well as chemical and gas sensors. Unusual properties such as valley polarization and a strong spin-orbit effect have also been demonstrated. In studying the physics and chemistry of $2 \mathrm{D}$ TMDCs, researchers will be able to draw upon the previous research on the bulk TMDCs' intercalation chemistry, materials processing and characterization, along with techniques for device fabrication and nanoscale characterization developed with carbon nanotubes and graphene. But 2D TMDCs have many distinctive properties that are not seen in other materials systems, and as researchers learn more about them, there are sure to be unexpected and exciting applications.

In the next few years, progress in this field will require advances in scalable and controllable sample preparation to make large amounts of atomically thin and uniform TMDC layers, either in solution or on substrates. For solution-phase preparation, the challenges include methods to control the area and thickness of either exfoliated or chemically grown flakes, and to find new methods and chemicals that can efficiently and safely produce these materials in large volumes. For solid-state samples, crystal growth methods need to improve to achieve large areas, large grain sizes, uniformity and control of layer number. Access to high-quality samples 
will enable more researchers to better understand the physical and chemical properties of TMDCs, as well as pursue a wide variety of applications. The properties and applications of 2D TDMC materials are a relatively new but exciting and rapidly expanding area of research.

\section{Received 11 July 2012; accepted 2 October 2012;} published online 6 November 2012.

\section{References}

1. Novoselov, K. S. et al. Two-dimensional atomic crystals. Proc. Natl Acad. Sci. USA 102, 10451-10453 (2005).

2. Castro Neto, A. H., Guinea, F., Peres, N. M. R., Novoselov, K. S. \& Geim, A. K. The electronic properties of graphene. Rev. Mod. Phys. 81, 109-162 (2009).

3. Geim, A. K. Graphene: status and prospects. Science 324, 1530-1534 (2009).

4. Balandin, A. A. et al. Superior thermal conductivity of single-layer graphene. Nano Lett. 8, 902-907 (2008).

5. Mayorov, A. S. et al. Micrometer-scale ballistic transport in encapsulated graphene at room temperature. Nano Lett. 11, 2396-2399 (2011).

6. Bunch, J. S. et al. Impermeable atomic membranes from graphene sheets. Nano Lett. 8, 2458-2462 (2008).

7. Nair, R. R., Wu, H. A., Jayaram, P. N., Grigorieva, I. V. \& Geim, A. K. Unimpeded permeation of water through helium-leak-tight graphene-based membranes. Science 335, 442-444 (2012).

8. Mattheis, L. F. Band structures of transition-metal-dichalcogenide layer compounds. Phys. Rev. B 8, 3719-3740 (1973).

9. Wilson, J. A. \& Yoffe, A. D. Transition metal dichalcogenides: discussion and interpretation of observed optical, electrical and structural properties. Adv. Phys. 18, 193-335 (1969).

10. Osada, M. \& Sasaki, T. Two-dimensional dielectric nanosheets: novel nanoelectronics from nanocrystal building blocks. Adv. Mater. 24, 210-228 (2012).

11. Ayari, A., Cobas, E., Ogundadegbe, O. \& Fuhrer, M. S. Realization and electrical characterization of ultrathin crystals of layered transition-metal dichalcogenides. J. Appl. Phys. 101, 014507 (2007).

12. Dean, C. R. et al. Boron nitride substrates for high-quality graphene electronics. Nature Nanotech. 5, 722-726 (2010).

13. Pacile, D., Meyer, J. C., Girit, C. O. \& Zettl, A. The two-dimensional phase of boron nitride: few-atomic-layer sheets and suspended membranes. Appl. Phys. Lett. 92, (2008).

14. Yoffe, A. D. Layer compounds. Annu. Rev. Mater. Sci. 3, 147-170 (1993).

15. Yoffe, A. D. Low-dimensional systems: quantum size effects and electronic properties of semiconductor microcrystallites (zero-dimensional systems) and some quasi-two-dimensional systems. Adv. Phys. 42, 173-266 (1993).

16. Elias, D. C. et al. Dirac cones reshaped by interaction effects in suspended graphene. Nature Phys. 7, 701-704 (2011).

17. Lin, M-W. et al. Room-temperature high on/off ratio in suspended graphene nanoribbon field-effect transistors. Nanotechnology 22, 265201 (2011).

18. Li, X., Wang, X., Zhang, L., Lee, S. \& Dai, H. Chemically derived, ultrasmooth graphene nanoribbon semiconductors. Science 319, 1229-1232 (2008).

19. Han, M. Y., Özyilmaz, B., Zhang, Y. \& Kim, P. Energy band-gap engineering of graphene nanoribbons. Phys. Rev. Lett. 98, 206805 (2007).

20. Balog, R. et al. Bandgap opening in graphene induced by patterned hydrogen adsorption. Nature Mater. 9, 315-319 (2010).

21. Zhang, Y. et al. Direct observation of a widely tunable bandgap in bilayer graphene. Nature 459, 820-823 (2009).

22. Sipos, B. et al. From Mott state to superconductivity in $1 \mathrm{~T}-\mathrm{TaS}_{2}$. Nature Mater. 7, 960-965 (2008).

23. Wilson, J. A., Disalvo, F. J. \& Mahajan, S. Charge-density waves and superlattices in metallic layered transition-metal dichalcogenides. Adv. Phys. 24, 117-201 (1975).

24. Castro Neto, A. H. Charge density wave, superconductivity, and anomalous metallic behavior in 2D transition metal dichalcogenides. Phys. Rev. Lett. 86, 4382-4385 (2001).

25. Kuc, A., Zibouche, N. \& Heine, T. Influence of quantum confinement on the electronic structure of the transition metal sulfide TS, Phys. Rev. B 83, 245213 (2011).

26. Mak, K. F., He, K., Shan, J. \& Heinz, T. F. Control of valley polarization in monolayer $\mathrm{MoS}_{2}$ by optical helicity. Nature Nanotech. 7, 494-498 (2012).

27. Zeng, H., Dai, J., Yao, W., Xiao, D. \& Cui, X. Valley polarization in $\mathrm{MoS}_{2}$ monolayers by optical pumping. Nature Nanotech. 7, 490-493 (2012).

28. Cao, T. et al. Valley-selective circular dichroism of monolayer molybdenum disulphide. Nature Commun. 3, 887 (2012).

29. Alem, N. et al. Atomically thin hexagonal boron nitride probed by ultrahighresolution transmission electron microscopy. Phys. Rev. B 80, 155425 (2009).
30. Lee, C. et al. Anomalous lattice vibrations of single- and few-layer $\mathrm{MoS}_{2}$. ACS Nano 4, 2695-2700 (2010).

31. Mak, K. F., Lee, C., Hone, J., Shan, J. \& Heinz, T. F. Atomically thin $\operatorname{MoS}_{2}: \mathrm{a}$ new direct-gap semiconductor. Phys. Rev. Lett. 105, 136805 (2010).

32. Splendiani, A. et al. Emerging photoluminescence in monolayer $\mathrm{MoS}_{2}$. Nano Lett. 10, 1271-1275 (2010)

33. Bertolazzi, S., Brivio, J. \& Kis, A. Stretching and breaking of ultrathin $\mathrm{MoS}_{2}$. ACS Nano 5, 9703-9709 (2011).

34. Radisavljevic, B., Radenovic, A., Brivio, J., Giacometti, V. \& Kis, A. Single-layer $\mathrm{MoS}_{2}$ transistors. Nature Nanotech. 6, 147-150 (2011).

35. Radisavljevic, B., Whitwick, M. B. \& Kis, A. Integrated circuits and logic operations based on single-layer $\mathrm{MoS}_{2}$. ACS Nano 5, 9934-9938 (2011).

36. Benameur, M. M. et al. Visibility of dichalcogenide nanolayers. Nanotechnology 22, 125706 (2011).

37. Li, H. et al. Optical identification of single- and few-layer $\mathrm{MoS}_{2}$ sheets. Small 8, 682-686 (2012)

38. Kalantar-Zadeh, K. et al. Synthesis of atomically thin $\mathrm{WO}_{3}$ sheets from hydrated tungsten trioxide. Chem. Mater. 22, 5660-5666 (2010).

39. Yin, Z. et al. Single-layer $\mathrm{MoS}_{2}$ phototransistors. ACS Nano 6, 74-80 (2012).

40. Feng, J. et al. Giant moisture responsiveness of $\mathrm{VS}_{2}$ ultrathin nanosheets for novel touchless positioning interface. Adv. Mater. 24, 1969-1974 (2012).

41. Zhang, Y., Ye, J., Matsuhashi, Y. \& Iwasa, Y. Ambipolar $\mathrm{MoS}_{2}$ thin flake transistors. Nano Lett. 12, 1136-1140 (2012).

42. Castellanos-Gomez, A. et al. Laser-thinning of $\mathrm{MoS}_{2}$ : on demand generation of a single-layer semiconductor. Nano Lett. 12, 3187-3192 (2012).

43. Coleman, J. N. et al. Two-dimensional nanosheets produced by liquid exfoliation of layered materials. Science 331, 568-571 (2011).

44. Smith, R. J. et al. Large-scale exfoliation of inorganic layered compounds in aqueous surfactant solutions. Adv. Mater. 23, 3944-3948 (2011).

45. Sire, C. d. et al. Flexible gigahertz transistors derived from solution-based single-layer graphene. Nano Lett. 12, 1184-1188 (2012).

46. Bissessur, R., Heising, J. \& Hirpo, W. Toward pillared layered metal sulfides. intercalation of the chalcogenide clusters $\mathrm{Co}_{6} \mathrm{Q}_{8}\left(\mathrm{PR}_{3}\right)_{6}(\mathrm{Q}=\mathrm{S}, \mathrm{Se}$, and Te and $\mathrm{R}=$ Alkyl) into $\mathrm{MoS}_{2}$. Chem. Mater. 8, 318-320 (1996).

47. Joensen, P., Frindt, R. F. \& Morrison, S. R. Single-layer $\mathrm{MoS}_{2}$. Mater. Res. Bull. 21, 457-461 (1986).

48. Osada, M. \& Sasaki, T. Exfoliated oxide nanosheets: new solution to nanoelectronics. J. Mater. Chem. 19, 2503-2511 (2009).

49. Eda, G. et al. Photoluminescence from chemically exfoliated $\mathrm{MoS}_{2}$. Nano Lett. 11, 5111-5116 (2011)

50. Zeng, Z. Y. et al. Single-layer semiconducting nanosheets: high-yield preparation and device fabrication. Angew. Chem. Int. Ed. 50, 11093-11097 (2011).

51. Dines, M. B. Lithium intercalation via n-butyllithium of layered transitionmetal dichalcogenides. Mater. Res. Bull. 10, 287-291 (1975).

52. Tsai, H-L., Heising, J., Schindler, J. L., Kannewurf, C. R. \& Kanatzidis, M. G. Exfoliated-restacked phase of $\mathrm{WS}_{2}$. Chem. Mater. 9, 879-882 (1997).

53. Frey, G. L., Reynolds, K. J., Friend, R. H., Cohen, H. \& Feldman, Y. Solution processed anodes from layer-structure materials for high-efficiency polymer light-emitting diodes. J. Am. Chem. Soc. 125, 5998-6007 (2003).

54. Bissessur, R., Kanatzidis, M. G., Schindler, J. L. \& Kannewurf, C. R. Encapsulation of polymers into $\mathrm{MoS}_{2}$ and metal to insulator transition in metastable $\mathrm{MoS}_{2}$. J. Chem. Soc. Chem. Commun. 1582-1585 (1993).

55. Gordon, R. A., Yang, D., Crozier, E. D., Jiang, D. T. \& Frindt, R. F. Structures of exfoliated single layers of $\mathrm{WS}_{2}, \mathrm{MoS}_{2}$, and $\mathrm{MoSe}_{2}$ in aqueous suspension. Phys. Rev. B 65, (2002)

56. Kirmayer, S., Aharon, E., Dovgolevsky, E., Kalina, M. \& Frey, G. L. Self-assembled lamellar $\mathrm{MoS}_{2}, \mathrm{SnS}_{2}$ and $\mathrm{SiO}_{2}$ semiconducting polymer nanocomposites. Phil. Trans. R. Soc. A 365, 1489-1508 (2007).

57. Zeng, Z. et al. An effective method for the fabrication of few-layer-thick inorganic nanosheets. Angew. Chem. Int. Ed. 51, 9052-9056 (2012).

58. Zhou, K-G., Mao, N-N., Wang, H-X., Peng, Y. \& Zhang, H-L. A mixedsolvent strategy for efficient exfoliation of inorganic graphene analogues. Angew. Chem. Int. Ed. 50, 10839-10842 (2011).

59. May, P., Khan, U., Hughes, J. M. \& Coleman, J. N. Role of solubility parameters in understanding the steric stabilization of exfoliated two-dimensional nanosheets by adsorbed polymers. J. Phys. Chem. C 116, 11393-11400 (2012).

60. Cunningham, G. et al. Solvent Exfoliation of transition metal dichalcogenides: dispersibility of exfoliated nanosheets varies only weakly between compounds. ACS Nano 6, 3468-3480 (2012).

61. Díaz, E., Ordóñez, S. \& Vega, A. Adsorption of volatile organic compounds onto carbon nanotubes, carbon nanofibers, and high-surface-area graphites. J. Colloid Interface Sci. 305, 7-16 (2007).

62. Hernandez, Y. et al. High-yield production of graphene by liquid-phase exfoliation of graphite. Nature Nanotech. 3, 563-568 (2008).

63. Shih, C-J. et al. Bi- and trilayer graphene solutions. Nature Nanotech. 6, 439-445 (2011). 
64. Green, A. A. \& Hersam, M. C. Solution phase production of graphene with controlled thickness via density differentiation. Nano Lett. 9, 4031-4036 (2009).

65. O’Neill, A., Khan, U. \& Coleman, J. N. Preparation of high concentration dispersions of exfoliated $\mathrm{MoS}_{2}$ with increased flake size. Chem. Mater. 24, 2414-2421 (2012)

66. Li, X. et al. Large-area synthesis of high-quality and uniform graphene films on copper foils. Science 324, 1312-1314 (2009).

67. Hass, J., de Heer, W. A. \& Conrad, E. H. The growth and morphology of epitaxial multilayer graphene. J. Phys. Condens. Matter 20, (2008)

68. Wu, Y. et al. State-of-the-art graphene high-frequency electronics. Nano Lett. 12, 3062-3067 (2012)

69. Wu, Y. et al. High-frequency, scaled graphene transistors on diamond-like carbon. Nature 472, 74-78 (2011).

70. Lin, Y-M. et al. Wafer-scale graphene integrated circuit. Science 332, 1294-1297 (2011).

71. Lee, Y-H. et al. Synthesis of large-area $\mathrm{MoS}_{2}$ atomic layers with chemical vapor deposition. Adv. Mater. 24, 2320-2325 (2012).

72. Zhan, Y., Liu, Z., Najmaei, S., Ajayan, P. M. \& Lou, J. Large-area vapor-phase growth and characterization of $\mathrm{MoS}_{2}$ atomic layers on a $\mathrm{SiO}_{2}$ substrate. Small 8, 966-971 (2012)

73. Liu, K.-K. et al. Growth of large-area and highly crystalline $\mathrm{MoS}_{2}$ thin layers on insulating substrates. Nano Lett. 12, 1538-1544 (2012).

74. Balendhran, S. et al. Atomically thin layers of $\mathrm{MoS}_{2}$ via a two step thermal evaporation-exfoliation method. Nanoscale 4, 461-466 (2012).

75. Shi, Y. et al. Van der Waals epitaxy of $\mathrm{MoS}_{2}$ layers using graphene as growth templates. Nano Lett. 12, 2784-2791 (2012).

76. Peng, Y. et al. Hydrothermal synthesis of $\mathrm{MoS}_{2}$ and its pressure-related crystallization. J. Solid State Chem. 159, 170-173 (2001).

77. Peng, Y. et al. Hydrothermal synthesis and characterization of singlemolecular-layer $\mathrm{MoS}_{2}$ and $\mathrm{MoSe}_{2}$. Chem. Lett. 30, 772-773 (2001).

78. Matte, H. S. S. et al. $\mathrm{MoS}_{2}$ and $\mathrm{WS}_{2}$ Analogues of graphene. Angew. Chem. Int. Ed. 49, 4059-4062 (2010).

79. Matte, H. S. S. R., Plowman, B., Datta, R. \& Rao, C. N. R. Graphene analogues of layered metal selenides. Dalton Trans. 40, 10322-10325 (2011).

80. Kobayashi, K. \& Yamauchi, J. Electronic structure and scanning-tunnelingmicroscopy image of molybdenum dichalcogenide surfaces. Phys. Rev. B 51, 17085-17095 (1995).

81. Li, T. \& Galli, G. Electronic properties of $\mathrm{MoS}_{2}$ nanoparticles. J. Phys. Chem. C 111, 16192-16196 (2007).

82. Liu, L., Kumar, S. B., Ouyang, Y. \& Guo, J. Performance limits of monolayer transition metal dichalcogenide transistors. IEEE Trans. Electron Devices 58, 3042-3047 (2011)

83. Ding, Y. et al. First principles study of structural, vibrational and electronic properties of graphene-like $\mathrm{MX}_{2}(\mathrm{M}=\mathrm{Mo}, \mathrm{Nb}, \mathrm{W}, \mathrm{Ta} ; \mathrm{X}=\mathrm{S}, \mathrm{Se}, \mathrm{Te})$ monolayers. Physica B 406, 2254-2260 (2011).

84. Ataca, C., Şahin, H. \& Ciraci, S. Stable, single-layer MX transition-metal oxides and dichalcogenides in a honeycomb-like structure. J. Phys. Chem. C 116, 8983-8999 (2012).

85. Lebègue, S. \& Eriksson, O. Electronic structure of two-dimensional crystals from ab initio theory. Phys. Rev. B 79, 115409 (2009).

86. Frindt, R. F. The optical properties of single crystals of $\mathrm{WSe}_{2}$ and $\mathrm{MoTe}_{2}$ J. Phys. Chem. Solids 24, 1107-1108 (1963).

87. Frindt, R. F. \& Yoffe, A. D. Physical properties of layer structures: optical properties and photoconductivity of thin crystals of molybdenum disulphide. Proc. R. Soc. Lond. A 273, 69-83 (1963).

88. Kam, K. K. \& Parkinson, B. A. Detailed photocurrent spectroscopy of the semiconducting group-VI transition-metal dichalcogenides. J. Phys. Chem. 86, 463-467 (1982).

89. Bollinger, M. V. et al. One-dimensional metallic edge states in $\mathrm{MoS}_{2}$. Phys. Rev. Lett. 87, 196803 (2001).

90. Böker, T. et al. Band structure of $\mathrm{MoS}_{2}, \mathrm{MoSe}_{2}$, and $\alpha-\mathrm{MoTe}_{2}$ : angle-resolved photoelectron spectroscopy and $a b$ initio calculations. Phys. Rev. B 64, 235305 (2001)

91. Schwierz, F. Graphene transistors. Nature Nanotech. 5, 487-496 (2010).

92. The International Technology Roadmap for Semiconductors. http://www.itrs.net/Links/2011ITRS/Home2011.htm (Semiconductor Industry Association, 2011)

93. Sze, S. M. \& Ng, K. K. Physics of Semiconductor Devices (Wiley, 2007).

94. Morkoc, H. et al. Large-band-gap SiC, III-V nitride, and II-VI ZnSe-based semiconductor device technologies. J. Appl. Phys. 76, 1363-1398 (1994).

95. Avouris, P., Chen, Z. \& Perebeinos, V. Carbon-based electronics. Nature Nanotech. 2, 605-615 (2007).

96. Avouris, P., Freitag, M. \& Perebeinos, V. Carbon-nanotube photonics and optoelectronics. Nature Photon. 2, 341-350 (2008).

97. Lu, W. \& Lieber, C. M. Nanoelectronics from the bottom up. Nature Mater. 6, 841-850 (2007)
98. Yoon, Y., Ganapathi, K. \& Salahuddin, S. How good can monolayer $\mathrm{MoS}_{2}$ transistors be? Nano Lett. 11, 3768-3773 (2011).

99. Colinge, J-P. Multiple-gate SOI MOSFETs. Solid State Electron. 48, 897-905 (2004).

100. Ando, T., Fowler, A. B. \& Stern, F. Electronic properties of two-dimensional systems. Rev. Mod. Phys. 54, 437-672 (1982).

101. Ridley, B. K. The electron-phonon interaction in quasi-two-dimensional semiconductor quantum-well structures. J. Phys. C 15, 5899 (1982)

102. Chen, J-H., Jang, C., Xiao, S., Ishigami, M. \& Fuhrer, M. S. Intrinsic and extrinsic performance limits of graphene devices on $\mathrm{SiO}_{2}$. Nature Nanotech. 3, 206-209 (2008)

103. Adam, S., Hwang, E. H. \& Das Sarma, S. Scattering mechanisms and Boltzmann transport in graphene. Physica E 40, 1022-1025 (2008).

104. Kaasbjerg, K., Thygesen, K. S. \& Jacobsen, K. W. Phonon-limited mobility in n-type single-layer $\mathrm{MoS}_{2}$ from first principles. Phys. Rev. B 85, 115317 (2012).

105. Hwang, E. H., Adam, S. \& Das Sarma, S. Carrier transport in two-dimensional graphene layers. Phys. Rev. Lett. 98, 186806 (2007).

106. Jena, D. \& Konar, A. Enhancement of carrier mobility in semiconductor nanostructures by dielectric engineering. Phys. Rev. Lett. 98, 136805 (2007)

107. Konar, A., Fang, T. \& Jena, D. Effect of high- $\kappa$ gate dielectrics on charge transport in graphene-based field effect transistors. Phys. Rev. B 82, 115452 (2010).

108. Ponomarenko, L. A. et al. Effect of a high- $\kappa$ environment on charge carrier mobility in graphene. Phys. Rev. Lett. 102, 206603 (2009).

109. Zhu, W., Perebeinos, V., Freitag, M. \& Avouris, P. Carrier scattering, mobilities, and electrostatic potential in monolayer, bilayer, and trilayer graphene. Phys. Rev. B 80, 235402 (2009).

110. Kim, S. et al. High-mobility and low-power thin-film transistors based on multilayer $\mathrm{MoS}_{2}$ crystals. Nature Commun. 3, 1011 (2012).

111. Sakaki, H., Noda, T., Hirakawa, K., Tanaka, M. \& Matsusue, T. Interface roughness scattering in GaAs/AlAs quantum wells. Appl. Phys. Lett. 51, 1934-1936 (1987).

112. Castro, E. V. et al. Limits on charge carrier mobility in suspended graphene due to flexural phonons. Phys. Rev. Lett. 105, 266601 (2010).

113. Brivio, J., Alexander, D. T. L. \& Kis, A. Ripples and layers in ultrathin $\mathrm{MoS}_{2}$ membranes. Nano Lett. 11, 5148-5153 (2011).

114. Fivaz, R. \& Mooser, E. Mobility of charge carriers in semiconducting layer structures. Phys. Rev. 163, 743-755 (1967).

115. Podzorov, V., Gershenson, M. E., Kloc, C., Zeis, R. \& Bucher, E. High-mobility field-effect transistors based on transition metal dichalcogenides. Appl. Phys. Lett. 84, 3301-3303 (2004)

116. Newaz, A. K. M., Puzyrev, Y. S., Wang, B., Pantelides, S. T. \& Bolotin, K. I. Probing charge scattering mechanisms in suspended graphene by varying its dielectric environment. Nature Commun. 3, 734 (2012).

117. Chen, F., Xia, J., Ferry, D. K. \& Tao, N. Dielectric screening enhanced performance in graphene FET. Nano Lett. 9, 2571-2574 (2009).

118. Fang, H. et al. High-performance single layered WSe $e_{2}$-FETs with chemically doped contacts. Nano Lett. 12, 3788-3792 (2012).

119. Lee, K. et al. Electrical characteristics of molybdenum disulfide flakes produced by liquid exfoliation. Adv. Mater. 23, 4178-4182 (2011).

120. Brown, S. \& Vranesic, Z. Fundamentals of Digital Logic with VHDL Design. (McGraw-Hill, 2008).

121. Wang, H. et al. Integrated circuits based on bilayer $\mathrm{MoS}_{2}$ transistors. Nano Lett. 12, 4674-4680 (2012).

122. He, Q. et al. Fabrication of flexible $\mathrm{MoS}_{2}$ thin-film transistor arrays for practical gas-sensing applications. Small 8, 2994-2999 (2012).

123. $\mathrm{Pu}$, J. et al. Highly flexible $\mathrm{MoS}_{2}$ thin-film transistors with ion gel dielectrics. Nano Lett. 12, 4013-4017 (2012).

124. Khan, M. A., Bhattarai, A., Kuznia, J. N. \& Olson, D. T. High electron mobility transistor based on a GaN- $\mathrm{Al}_{x} \mathrm{Ga}_{1-x} \mathrm{~N}$ heterojunction. Appl. Phys. Lett. 63, 1214-1215 (1993).

125. Britnell, L. et al. Field-effect tunneling transistor based on vertical graphene heterostructures. Science 335, 947-950 (2012).

126. Scholes, G. D. \& Rumbles, G. Excitons in nanoscale systems. Nature Mater 5, 683-696 (2006).

127. Kamat, P. V. Quantum dot solar cells. Semiconductor nanocrystals as light harvesters. J. Phys. Chem. C 112, 18737-18753 (2008).

128. Law, M., Goldberger, J. \& Yang, P. D. Semiconductor nanowires and nanotubes. Annu. Rev. Mater. Res. 34, 83-122 (2004).

129. Coehoorn, R., Haas, C. \& de Groot, R. A. Electronic structure of $\mathrm{MoSe}_{2}, \mathrm{MoS}_{2}$ and $\mathrm{WSe}_{2}$. II. The nature of the optical band gaps. Phys. Rev. B 35, 6203-6206 (1987).

130. Ramasubramaniam, A. Large excitonic effects in monolayers of molybdenum and tungsten dichalcogenides. Phys. Rev. B 86, 115409 (2012).

131. Cheiwchanchamnangij, T. \& Lambrecht, W. R. L. Quasiparticle band structure calculation of monolayer, bilayer, and bulk $\mathrm{MoS}_{2}$. Phys. Rev. B 85, 205302 (2012) 
132. Molina-Sanchez, A. \& Wirtz, L. Phonons in single-layer and few-layer $\mathrm{MoS}_{2}$ and $\mathrm{WS}_{2}$. Phys. Rev. B 84, 155413 (2011)

133. Nair, R. R. et al. Fine structure constant defines visual transparency of graphene. Science 320, 1308 (2008).

134. Bae, S. et al. Roll-to-roll production of 30 -inch graphene films for transparent electrodes. Nature Nanotech. 5, 574-578 (2010).

135. Bonaccorso, F., Sun, Z., Hasan, T. \& Ferrari, A. C. Graphene photonics and optoelectronics. Nature Photon. 4, 611-622 (2010).

136. Wang, X., Zhi, L. \& Mullen, K. Transparent, conductive graphene electrodes for dye-sensitized solar cells. Nano Lett. 8, 323-327 (2007).

137. Alharbi, F. et al. Abundant non-toxic materials for thin film solar cells: alternative to conventional materials. Renew. Energy 36, 2753-2758 (2011).

138. Beal, A. R., Hughes, H. P. \& Liang, W. Y. The reflectivity spectra of some group VA transition metal dichalcogenides. J. Phys. C 8, 4236 (1975).

139. Chandra, S., Singh, D. P., Srivastava, P. C. \& Sahu, S. N. Electrodeposited semiconducting molybdenum selenide films. II. Optical, electrical, electrochemical and photoelectrochemical solar cell studies. J. Phys. D: Appl. Phys. 17, 2125 (1984).

140. Shimada, T. et al. Work function and photothreshold of layered metal dichalcogenides. Jpn. J. Appl. Phys. 33, 2696.

141. Friend, R. H. \& Yoffe, A. D. Electronic-properties of intercalation complexes of the transition-metal dichalcogenides. Adv. Phys. 36, 1-94 (1987).

142. Benavente, E., Santa Ana, M. A., Mendizabal, F. \& Gonzalez, G. Intercalation chemistry of molybdenum disulfide. Coord. Chem. Rev. 224, 87-109 (2002).

143. Gourmelon, E. et al. $\mathrm{MS}_{2}(\mathrm{M}=\mathrm{W}, \mathrm{Mo})$ photosensitive thin films for solar cells. Sol. Energ. Mater. Sol. Cells 46, 115-121 (1997).

144. Lee, H. S. et al. $\mathrm{MoS}_{2}$ nanosheet phototransistors with thickness-modulated optical energy gap. Nano Lett. 12, 446-701 (2012).

145. Shanmugam, M., Bansal, T., Durcan, C. A. \& Yu, B. Molybdenum disulphide/ titanium dioxide nanocomposite-poly 3-hexylthiophene bulk heterojunction solar cell. Appl. Phys. Lett. 100, 153901-153904 (2012).

146. Thomalla, M. \& Tributsch, H. Photosensitization of nanostructured $\mathrm{TiO}_{2}$ with $\mathrm{WS}_{2}$ quantum sheets. J. Phys. Chem. B 110, 12167-12171 (2006).

147. Ho, W., Yu, J. C., Lin, J., Yu, J. \& Li, P. Preparation and photocatalytic behavior of $\mathrm{MoS}_{2}$ and $\mathrm{WS}_{2}$ nanocluster sensitized $\mathrm{TiO}_{2}$. Langmuir 20, 5865-5869 (2004)

148. Reynolds, K. J., Barker, J. A., Greenham, N. C., Friend, R. H. \& Frey, G. L. Inorganic solution-processed hole-injecting and electron-blocking layers in polymer light-emitting diodes. J. Appl. Phys. 92, 7556-7563 (2002).

149. Polman, A. \& Atwater, H. A. Photonic design principles for ultrahighefficiency photovoltaics. Nature Mater. 11, 174-177 (2012).

150. Gokus, T. et al. Making graphene luminescent by oxygen plasma treatment. ACS Nano 3, 3963-3968 (2009).
151. Eda, G. et al. Blue photoluminescence from chemically derived graphene oxide. Adv. Mater. 22, 505-509 (2010).

152. Carladous, A. et al. Light emission from spectral analysis of $\mathrm{Au} / \mathrm{MoS}_{2}$ nanocontacts stimulated by scanning tunneling microscopy. Phys. Rev. B 66, 045401 (2002).

153. Xiao, D., Liu, G.-B., Feng, W., Xu, X. \& Yao, W. Coupled spin and valley physics in monolayers of $\mathrm{MoS}_{2}$ and other group-VI dichalcogenides. Phys. Rev. Lett. 108, 196802 (2012).

154. Zhu, Z. Y., Cheng, Y. C. \& Schwingenschlögl, U. Giant spin-orbit-induced spin splitting in two-dimensional transition-metal dichalcogenide semiconductors. Phys. Rev. B 84, 153402 (2011).

155. Rycerz, A., Tworzydlo, J. \& Beenakker, C. W. J. Valley filter and valley valve in graphene. Nature Phys. 3, 172-175 (2007).

156. Xiao, D., Yao, W. \& Niu, Q. Valley-contrasting physics in graphene: magnetic moment and topological transport. Phys. Rev. Lett. 99, 236809 (2007).

157. Schedin, F. et al. Detection of individual gas molecules adsorbed on graphene. Nature Mater. 6, 652-655 (2007)

158. Fowler, J. D. et al. Practical chemical sensors from chemically derived graphene. ACS Nano 3, 301-306 (2009).

159. Dan, Y. P., Lu, Y., Kybert, N. J., Luo, Z. T. \& Johnson, A. T. C. Intrinsic response of graphene vapor sensors. Nano Lett. 9, 1472-1475 (2009).

160. Julien, C., Sekine, T. \& Balkanski, M. Lattice dynamics of lithium intercalated $\mathrm{MoS}_{2}$. Solid State Ionics 48, 225-229 (1991).

161. Li, H. et al. Fabrication of single- and multilayer $\mathrm{MoS}_{2}$ film-based field-effect transistors for sensing NO at room temperature. Small 8, 63-67 (2012).

162. Late, D. J., Liu, B., Matte, H. S. S. R., Dravid, V. P. \& Rao, C. N. R. Hysteresis in single-layer $\mathrm{MoS}_{2}$ field effect transistors. ACS Nano 6, 5635-5641 (2012).

163. Wu, S. et al. Electrochemically reduced single-layer $\mathrm{MoS}_{2}$ nanosheets: characterization, properties, and sensing applications. Small 8, 2264-2270 (2012)

164. Wilson, J. A., Di Salvo, F. J. \& Mahajan, S. Charge-density waves and superlattices in the metallic layered transition metal dichalcogenides. Adv. Phys. 24, 117-201 (1975).

165. Gmelin Handbook of Inorganic and Organometallic Chemistry 8th edn, Vol. B7 (Springer, 1995).

\section{Additional information}

Reprints and permissions information is available online at http://www.nature.com/ reprints. Correspondence should be addressed to M.S.S

\section{Competing financial interests}

The authors declare no competing financial interests. 